\title{
A parametric study on creep-fatigue strength of welded joints using the linear matching method
}

\author{
Yevgen Gorash, Haofeng Chen* \\ Department of Mechanical E Aerospace Engineering, University of Strathclyde, James Weir Building, 75 Montrose Street, Glasgow G1 1XJ, UK
}

\begin{abstract}
This paper presents a parametric study on creep-fatigue strength of the steel AISI type 316N(L) weldments of types 1 and 2 according to R5 Vol. 2/3 Procedure classification at $550^{\circ} \mathrm{C}$. The study is implemented using the Linear Matching Method (LMM) and is based upon a latest developed creep-fatigue evaluation procedure considering time fraction rule for creep-damage assessment. Parametric models of geometry and FE-meshes for both types of weldments are developed in this way, which allows variation of parameters governing shape of the weld profile and loading conditions. Five configurations, characterised by individual sets of parameters, and presenting different fabrication cases, are proposed. For each configuration, the total number of cycles to failure $N^{\star}$ in creep-fatigue conditions is assessed numerically for different loading cases including normalised bending moment $\tilde{M}$ and dwell period $\Delta t$. The obtained set of $N^{\star}$ is extrapolated by the analytic function, which is dependent on $\tilde{M}, \Delta t$ and geometrical parameters $\left(\alpha\right.$ and $\beta$ ). Proposed function for $N^{\star}$ shows good agreement with numerical results obtained by the LMM. Thus, it is used for the identification of Fatigue Strength Reduction Factors (FSRFs) intended for design purposes and dependent on $\Delta t, \alpha, \beta$.
\end{abstract}

Keywords: Creep, Damage, Finite element analysis, FSRF, Low-cycle fatigue, Type 316 steel, Weldment

\section{Introduction}

According to industrial experience, during the service life of welded structures subjected to cyclic loading at high temperature, welded joints are usually considered as the critical locations of potential creep-fatigue failure. This is caused by higher stress concentration, altered and non-uniform material properties of weldments compared to the parent material of the entire structure. Therefore, creep and fatigue characteristics of welded joints are of a priority importance for long-term integrity assessments and design of welded structures. There were attempts to develop analytical tools [1] to estimate longterm strength of welded joints under variable loading. However, residual life assessments are frequently complicated and inaccurate because of complex material microstructure and too many parameters affecting the strength of welded joints. They include technological parameters of welding process and postweld heat treatment, accuracy of modelling of weldment material microstructure, influence of residual stresses and distortions, geometrical parameters of the shape of the weld profile and non-welded root gaps, parameters of service conditions such as temperature, mechanical loading and dwell period. In view of the complexity of a unified model development for the assessment of creep-fatigue strength, there are a limited number of existing analytical approaches, but none of which are able to account for all of weldment parameters mentioned above. Thus,

*Corresponding author. Tel.: +44 141 5482036; Fax: +44 1415525105.

Email address: haofeng.chen@strath.ac.uk (Haofeng Chen)

URL: http://www.thelmm.co.uk (Haofeng Chen) long-term strength of weldments is a wide research area, which requires some unified integral approach able to improve the life prediction capability for welded joints. The most comprehensive overviews of studies devoted to investigation of influence of various parameters on fatigue life of welded joints are presented in $[1,2,3]$. However, the influence of creep on residual life is not investigated in these works.

This paper presents further extension of a latest developed approach [4], which includes a creep-fatigue evaluation procedure considering time fraction rule for creep-damage assessment and a recent revision of the Linear Matching Method (LMM) to perform a cyclic creep assessment [5]. The applicability of this approach to a creep-fatigue analysis was verified in [4] by the comparison of FEA/LMM predictions for an AISI type $316 \mathrm{~N}(\mathrm{~L})$ steel cruciform weldment at $550^{\circ} \mathrm{C}$ with experiments by Bretherton et al. [6, 7, 8, 9] with the overall objective of identifying fatigue strength reduction factors (FSRF) of austenitic weldments for further design applications. An overview of previous modelling studies devoted to analysis and simulation of these experiments [6, 7, 8, 9] is given in [4]. Generally they investigated an accuracy of residual life assessments according to R5 creep-fatigue crack initiation procedure [10] and its more recent revisions and potential improvements.

Effective and fast modelling of structural components with complex microstructure and material behaviour such as weldments under high-temperature and variable loading conditions can be implemented by the application of FEA with direct analysis methods, which calculate the stabilised cyclic response of structures with far less computational effort than full step-bystep analysis. The most practical among these methods are Di- 
rect Cyclic Analysis [11, 12] and the LMM framework [13, 14]. The LMM is distinguished from the other simplified methods by ensuring that both the equilibrium and compatibility are satisfied at each stage $[13,14,15,16]$. In addition to the shakedown analysis method [15], the LMM has been extended beyond the range of most other direct methods by including the evaluation of the ratchet limit $[13,14,16]$ and steady-state cyclic behaviour with creep-fatigue interaction $[17,18]$. The LMM ABAQUS user subroutines [19] have been consolidated by the R5 Procedure [10] research programme of EDF Energy to the commercial standard, and are counted to be the method most amenable to practical engineering applications involving complicated thermo-mechanical load history $[14,16]$. Following this, the LMM was much improved both theoretically and numerically [5] to include more accurate predictions of the stabilised cyclic response of a structure under creep-fatigue conditions. This, in turn, allowed more accurate assessments of the resulting cyclic and residual stresses, creep strain, plastic strain range, ratchet strain and elastic follow-up factor. Finally, to aid wider adoption of the LMM as an analysis tool for industry, the development of an Abaqus/CAE plug-in with GUI has been started [20]. For this purpose, the UMAT subroutine code has been significantly updated [20] to allow use of multi-processors for the FE-calculations of shakedown and ratchet limits.

The parametric study presented in this paper is based on the research outcomes given in prior work [4] validated by matching the basic experiments $[6,7,8,9]$. These outcomes briefly include: 1) more realistic modelling of a material behaviour of the weld regions (including LCF and creep endurance) when compared to previous studies; 2) a creep-fatigue evaluation procedure considering time fraction rule for creep-damage assessment and a non-linear creep-fatigue interaction diagram; 3) application of the recent revision of the LMM outlined in [5]. As a result, the approach proposed in [4] provides the most accurate numerical prediction of the experiments $[6,7,8,9]$ with less conservatism when compared to previous works, particularly to [18]. Thus, exactly the same assessment approach is used in the current study and is applied to parametric studies of the weldment geometry in order to assess the effect on the predicted life.

Another outcome of the previous work [4] is the formulation of an analytical function for the total number of cycles to failure $N^{\star}$ in creep-fatigue conditions, which is dependent on normalised bending moment $\tilde{M}$ and dwell period $\Delta t$. This function $N^{\star}(\tilde{M}, \Delta t)$ matches the LMM predictions with reasonable accuracy and is used for the investigation of $\Delta t$ influence on the FSRF. Therefore, the effect of creep on long-term strength of type 2 dressed weldments (according to the classification in R5 Vol. 2/3 Procedure [10]) is taken in to account.

Apart from accounting for operational parameters $(\tilde{M}$ and $\Delta t$ ), it is necessary to investigate the influence of a weld profile geometry on creep-fatigue strength within a parametric study. The introduction of geometrical parameters into the function $N^{\star}(\tilde{M}, \Delta t)$ allows the calculation of the FSRF as a continuous function able to cover a variety of weld profile geometries including type 1 and 2 in dressed, as-welded and intermediate configurations.

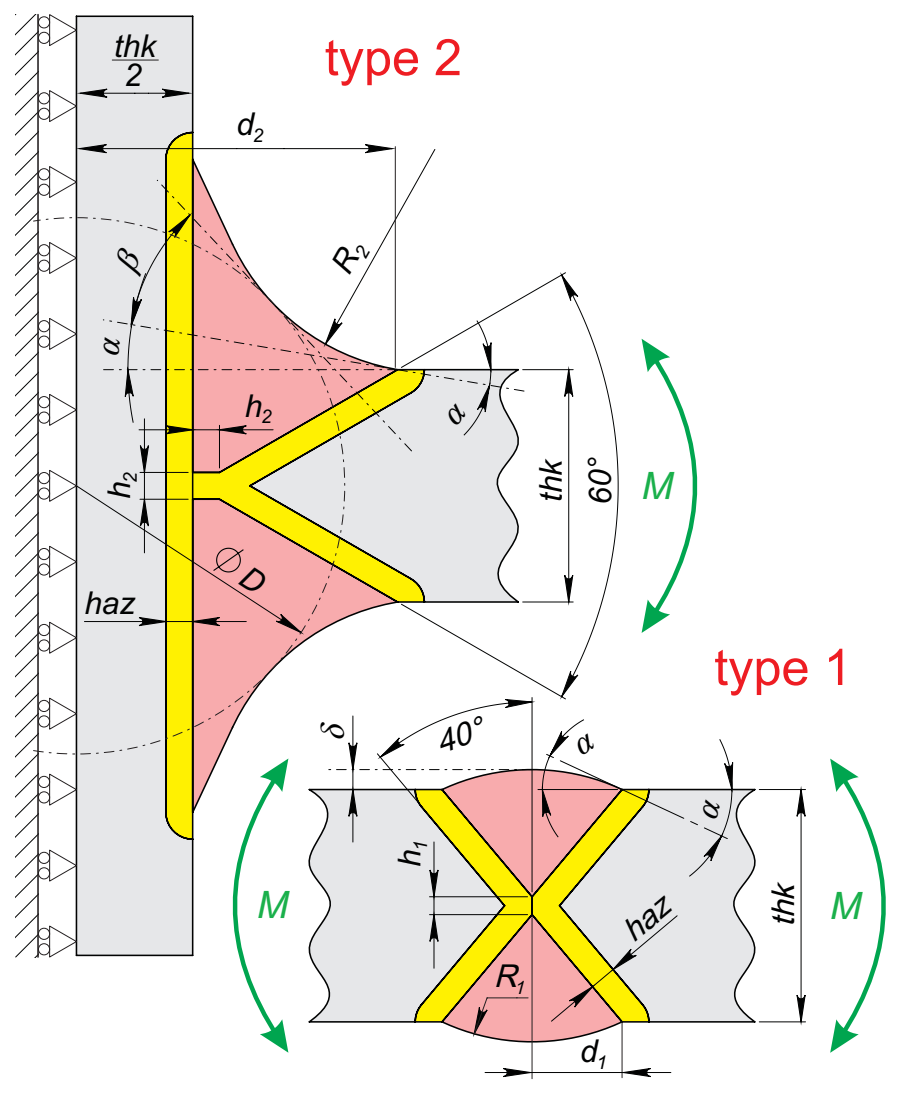

Figure 1: Designations of parameters fully describing weld profile geometries of types 1 and 2 weldments and applied bending moment, according to [6]

\section{Parametric models of weldments}

Referring to [1], generally creep-fatigue test results of weldment specimens contain various levels of scatter, which is usually caused by geometric and processing variations such as part fit-up, weld gap, variation in feed rates, travel rates, weld angles, etc. This scatter complicates the interpretation of test results, and often makes it nearly impossible to differentiate the effects of geometry, material non-uniformity, residual stress and other factors. It has been indicated [1] that one of the most critical factors affecting the creep-fatigue life of a welded joint is the consistency of the cross-sectional weld geometry. The simplified weld profile is usually characterised by the following geometric parameters [1]: plate thickness, effective weld throat thickness, weld leg length, weld throat angle, and weld toe radius. In this case the weld profile is assumed to be circular for type 1 and triangular for type 2 weldments with fillets on toes connecting with parent plates. A vast quantity of research reviewed in $[1,2,3]$ has been devoted to investigation of effects produced by these parameters on residual life.

In the present study, the geometry of the weld profile for type 2 weldment is more completely specified in order to investigate its as-welded, dressed and intermediate configurations. The basis of the parametric models shown in Fig. 1 are the sketches of the weldment specimens produced by the Manual Metal Arc (MMA) welding and reported in [6]. The type 1 weldment specimen contains a double-sided V-butt weld, and the type 2 weld- 
ment specimen contains 2 symmetric double-sided T-butt cruciform fillet welds. The parent material for the manufacturing of all specimens are continuous plates of width $w=200 \mathrm{~mm}$ and thickness $t h k=26 \mathrm{~mm}$ made of the steel type AISI 316N(L). The typical division of the weld into three regions is adopted here analogically to [4] including: parent material, weld metal and heat-affected zone (HAZ). It should be noted that the HAZ thickness is assumed to be $3 \mathrm{~mm}$ based on the geometry given in [6]. These 3 regions have different mechanical properties described by the following material behaviour models and corresponding constants at $550^{\circ} \mathrm{C}$ in [4] for the FEA with the LMM:

- Elastic-perfectly-plastic (EPP) model for the design limits as a result of shakedown analysis;

- Ramberg-Osgood (R-O) model for the plastic and total strains under saturated cyclic conditions;

- S-N diagrams for the number of cycles to failure caused by pure low-cycle fatigue (LCF);

- Power-law model in "time hardening" form for creep strains during primary creep stage;

- Reverse power-law relation for the time to creep rupture caused by creep relaxation during dwells;

- Non-linear diagrams for creep-fatigue damage interaction for the estimation of total damage.

The profile geometry of type 2 weldment is comprehensively characterised by one of two pairs of parameters: (1) independent parameters ( $\alpha$ and $\beta$ ), which are not dependent on a plate thickness $t h k$, and (2) technologically controlled parameters $\left(R_{2}\right.$ and $D$ ), which change their values with a change of plate thickness thk. The advantage of the 1 st couple is that it is not sensitive to simple scale transformation of the weldment geometry. The advantage of the 2 nd couple is that it could be easily measured and controlled according to technological requirements. Therefore, in parametric relations for strength of type 2 weldments the independent parameters ( $\alpha$ and $\beta$ ) should be used with a capability of transformation into controlled parameters $\left(R_{2}\right.$ and $\left.D\right)$. As illustrated in Fig. 1, angle $\alpha$ represents a local geometrical non-uniformity caused by a deviation from the tangent condition between parent plate and weld. Angle $\beta$ represents a global geometrical non-uniformity caused by deposition of weld metal connecting the orthogonal part.

The relations between the two parameter pairs ( $\alpha, \beta$ and $R_{2}$, $D$ ) for a type 2 weldment are formulated using basic trigonometric calculus in conjunction with the thickness of a plate cross-section thk and the corresponding associated parameters $\left(h_{2}\right.$ and $\left.d_{2}\right)$ as illustrated in Fig. 1:

$$
h_{2}=\frac{t h k}{8.6666} \text { and } d_{2}=\frac{t h k}{2}+h_{2}+\frac{t h k-h_{2}}{2} \tan 60^{\circ} \text {. }
$$

The direct transitions are formulated as follows

$$
\begin{aligned}
& R_{2}=\frac{\frac{t h k / 2}{\cos (\alpha+\beta)}-\frac{d_{2}}{\sin (\alpha+\beta)}}{\frac{\sin \alpha}{\sin (\alpha+\beta)}-\frac{\cos \alpha}{\cos (\alpha+\beta)}} \text { and } \\
& D=2 \frac{R_{2} \cos \alpha+t h k / 2}{\cos (\alpha+\beta)}-2 R_{2} .
\end{aligned}
$$

The reverse transitions are formulated as follows

$$
\begin{aligned}
\beta & =\arccos \left[\frac{d_{2}^{2}+(t h k / 2)^{2}-R_{2}^{2}-\left(R_{2}+D / 2\right)^{2}}{-2 R_{2}\left(R_{2}+D / 2\right)}\right], \\
\alpha & =90^{\circ}-\arctan \left(\frac{t h k}{2 d_{2}}\right)-\beta \\
& -\arccos \left[\frac{R_{2}^{2}-\left(R_{2}+D / 2\right)^{2}-d_{2}^{2}-(t h k / 2)^{2}}{-2\left(R_{2}+D / 2\right) \sqrt{d_{2}^{2}-(t h k / 2)^{2}}}\right] .
\end{aligned}
$$

Relations between independent parameter $\alpha$ and controlled parameter $\delta$ for type 1 weldment are formulated using basic trigonometric calculus in conjunction with the thickness of a plate cross-section thk and the corresponding associated parameters $\left(h_{1}\right.$ and $\left.d_{1}\right)$ as illustrated in Fig. 1:

$$
h_{1}=\frac{t h k}{13} \text { and } d_{1}=\frac{t h k-h_{1}}{2} \tan 40^{\circ} .
$$

The direct transition is formulated as follows

$$
\delta=R_{1}(1-\cos \alpha) \quad \text { with } \quad R_{1}=d_{1} / \sin \alpha .
$$

The reverse transition is formulated as follows

$$
\alpha=\arccos \left(\frac{R_{1}-\delta}{R_{1}}\right) \text { with } R_{1}=\frac{\delta}{2}+\frac{d_{1}^{2}}{2 \delta} .
$$

Since the proposed parameters for both types of weld profile are fully convertible, they can be used to characterise different scales of technological dressing of weldments by grinding such as dressed, as-welded and intermediate. Thus, in order to reduce the computational costs, only five configurations of weld profile, listed in Table 1, were chosen for parametric study from among the possible parameter combinations. It should be noted that configuration no. 2 of the type 2 weldment titled "typically dressed" (characterised in Fig. 1 by $h_{2}=3 \mathrm{~mm}, R_{2}=25 \mathrm{~mm}$, $D=59 \mathrm{~mm}, \alpha=7.745^{\circ}$ and $\beta=38.382^{\circ}$ ) has been an object of research in prior work [4]. Configuration no. 1 is characterised by a tangent condition between parent plate and weld profile contours. Configuration no. 5 presents the extreme variant of a roughly manufactured welded joint without any dressing. Thus, configurations no. 2, 3 and 4 correspond to some intermediate variants of weldment fabrication between the scales "perfectly dressed" and "coarsely as-welded".

The FE-meshes for the 2D symmetric models of type 1 and 2 weldments are shown in Fig. 2 assuming plane strain conditions. Each of the FE-meshes includes 5 separate areas with different material properties: 1) parent material, 2) HAZ, 3) weld metal, 4) material without creep, 5) totally elastic material. Introduction of 2 additional material types (material without creep 
Table 1: Geometrical configurations of weld profiles for type 1 and 2 weldments defined by the dimensions from Fig. 1

\begin{tabular}{cl|ccc|ccc}
\hline \hline \multirow{2}{*}{ No. } & \multirow{2}{*}{ Configuration } & \multicolumn{3}{|c|}{ Independent parameters } & \multicolumn{3}{|c}{ Controlled parameters } \\
\cline { 3 - 8 } & & $\alpha$ & $\beta$ & $\alpha+\beta$ & $D$ & $R$ & $\delta$ \\
\hline 1 & Perfectly dressed & 0 & 43.387 & 43.387 & 54.578 & 25 & 0 \\
2 & Typically dressed & 7.745 & 38.382 & 46.127 & 59 & 25 & 0.682 \\
3 & Precisely as-welded & 17.685 & 32.079 & 49.764 & 64 & 25 & 1.566 \\
4 & Typically as-welded & 32.371 & 18.415 & 50.786 & 68 & 40 & 2.923 \\
5 & Coarsely as-welded & 45.177 & 9.6541 & 54.831 & 72 & 60 & 4.189 \\
\hline \hline
\end{tabular}

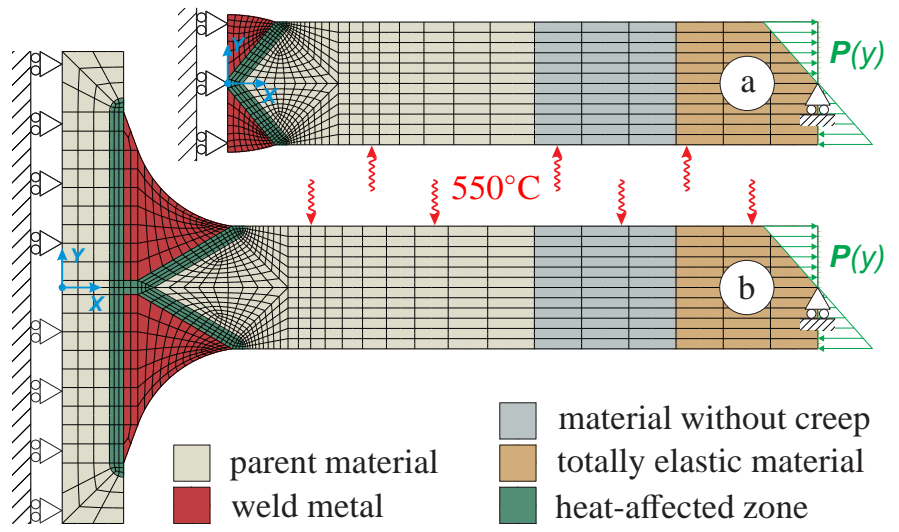

Figure 2: FE-meshes for type 1 (a) and type 2 (b) weldments with designation of different materials, boundary conditions and mechanical loading

and totally elastic material) representing reduced sets of parent material properties in the location of bending moment application avoids excessive stress concentrations in ratcheting and creep analysis. Both FE-models use ABAQUS element type CPE8R: 8-node biquadratic plane strain quadrilaterals with reduced integration. The FE-meshes for type 1 and type 2 welds consist of 723 and 977 elements respectively.

Referring to the technical details $[6,7,8,9]$ the testing was performed at $550 \pm 3^{\circ} \mathrm{C}$ under fully-reversed 4-point bending with total strain ranges $\Delta \varepsilon_{\text {tot }}$ of $0.25,0.3,0.4,0.6$ or $1.0 \%$ in the parent plate and hold periods $\Delta t$ of 0,1 or 5 hours using a strain rate of $0.03 \% / \mathrm{s}$. For the purpose of shakedown and creep analysis using LMM, the conversion from strain-controlled test conditions to force-controlled loading in the simulations using bending moment $M$ has been carried out and explained in [4].

Another effective analysis technique, successfully employed in [4], was to apply the bending moment $M$ through the linear distribution of normal pressure $P$ over the section of the plate as illustrated in Fig. 2 with the area moment of inertia in regard to horizontal axis $X$ :

$$
I_{X}=w t h k^{3} / 12,
$$

where the width of plate $w=200 \mathrm{~mm}$ and the thickness of plate thk $=26 \mathrm{~mm}$. Therefore, the normal pressure is expressed in terms of applied bending moment $M$ and vertical coordinate $y$ of plate section assuming the coordinate origin in the midsurface:

$$
P(y)=M y / I_{X} .
$$

\section{Plastic bending of plates}

\subsection{Solution with Ramberg-Osgood model}

The cyclic stress-strain properties of the steel AISI type $316 \mathrm{~N}(\mathrm{~L})$ parent material and associated weld and HAZ metals are presented in terms of the conventional Ramberg-Osgood equation and implemented in the LMM code for the creepfatigue analysis [4]. The R-O model has the advantage that it can be used to accurately represent the stress-strain curves of metals that harden with plastic deformation, showing a smooth elastic-plastic transition at high temperatures:

$$
\frac{\Delta \varepsilon_{\text {tot }}}{2}=\frac{\Delta \sigma}{2 \bar{E}}+\left(\frac{\Delta \sigma}{2 B}\right)^{1 / \beta},
$$

where $\Delta \varepsilon_{\text {tot }}$ is the total strain range; $\Delta \sigma$ is the equivalent stress range in $\mathrm{MPa} ; B$ and $\beta$ are plastic material constants; $\bar{E}$ is the effective elastic modulus in MPa defined as

$$
\bar{E}=\frac{3 E}{2(1+v)},
$$

where the Young's modulus $E$ in MPa and the Poisson's ratio $v$ are the uni-axial elastic material properties.

Although this relationship (9) is not explicitly solvable for stress range $\Delta \sigma$, an approximate solution for $\Delta \sigma$ can be found using following recursive formulation:

$$
\frac{\Delta \sigma_{n+1}}{2}=B\left(\frac{\Delta \varepsilon_{\text {tot }}}{2}-\frac{\Delta \sigma_{n}}{2 \bar{E}}\right)^{\beta} \quad \text { with } \quad n \geq 3,
$$

where the initial iteration is defined as

$$
\frac{\Delta \sigma_{0}}{2}=\left(\frac{\Delta \varepsilon_{\mathrm{tot}}}{2}\right)^{\beta} \text {. }
$$

For the case of plastic bending of a plate with a rectangular cross-section, i.e. as was used in the experimental studies implemented by Bretherton et al. [6, 7, 8, 9], it is possible to formulate an analytic relation using the R-O material model for the applied bending moment $M$ as proposed in [21]:

$$
M=\frac{2 w \sigma_{\text {eop }}}{3}\left(\frac{t h k}{2}\right)^{2}\left[\frac{1+\frac{3 \beta+3}{2 \beta+1} \tilde{\varepsilon}+\frac{3}{\beta+2} \tilde{\varepsilon}^{2}}{(1+\tilde{\varepsilon})^{2}}\right],
$$

where the maximum normal stress over a cross-section or edgeof-plate stress $\sigma_{\text {eop }}$ is defined based upon the plane strain assumption using equivalent stress $\sigma$

$$
\sigma_{\text {eop }}=2 \sigma / \sqrt{3}=\Delta \sigma / \sqrt{3}
$$


Table 2: The values of bending moment $M$ obtained by Eqs (11-15) corresponding to the values of total strain range $\Delta \varepsilon_{\text {tot }}$ from experiments $[6,7,8,9]$

\begin{tabular}{c|ccccc}
\hline \hline$\Delta \varepsilon_{\text {tot }}, \%$ & 1.0 & 0.6 & 0.4 & 0.3 & 0.25 \\
\hline$M, \mathrm{kN} \cdot \mathrm{m}$ & 10.068 & 7.924 & 6.368 & 5.347 & 4.739 \\
\hline \hline
\end{tabular}

and the ratio between plastic and elastic strains is formulated as

$$
\tilde{\varepsilon}=\frac{\varepsilon_{\mathrm{pl}}}{\varepsilon_{\mathrm{el}}}=\left(\frac{\Delta \sigma}{2 B}\right)^{1 / \beta} \frac{2 \bar{E}}{\Delta \sigma} .
$$

Other parameters of relation (13) include the material constants of the R-O model $(\beta, B, \bar{E})$ and the geometric parameters of a plate (thk and $w$ ). For the case of reverse bending tests of cruciform weldments at $550^{\circ} \mathrm{C}$ implemented by Bretherton et al. $[6,7,8,9]$, the total strain range $\Delta \varepsilon_{\text {tot }}$ in outer fibre of parent material plate remote from weld was controlled to correspond to one of the required values. Knowledge of the stabilised cycle parent material properties of the steel AISI type $316 \mathrm{~N}(\mathrm{~L})$ described by the R-O model (9) reported in Table 1 of [4] and geometric parameters of specimen $(t h k=26 \mathrm{~mm}$ and $w=200$ $\mathrm{mm}$ ) allows the calculation of the values of bending moments applied in experiments $[6,7,8,9]$ during the period of saturated cyclic response, as reported in Table 2.

Referring to [21], Eq. (13) gives a smooth variation of moment with strain, which could be derived analytically employing recursive formulas (11) and (12) for $\Delta \sigma$ dependent on $\Delta \varepsilon_{\text {tot }}$. Applying the recursive approach, the dependence of total strain range $\Delta \varepsilon_{\text {tot }}$ on applied moment $M$ could be obtained. Firstly, Eq. (13) is inverted to recursive formula taking into account Eq. (14) as follows:

$$
\begin{aligned}
& \frac{\Delta \sigma_{n+1}}{2}=\frac{M}{\frac{4 w}{3 \sqrt{3}}\left(\frac{t h k}{2}\right)^{2}\left[\frac{1+\frac{3 \beta+3}{2 \beta+1} \tilde{\varepsilon}_{n}+\frac{3}{\beta+2} \tilde{\varepsilon}_{n}^{2}}{\left(1+\tilde{\varepsilon}_{n}\right)^{2}}\right]} \\
& \text { with } \tilde{\varepsilon}_{n}=\left(\frac{\Delta \sigma_{n}}{2 B}\right)^{1 / \beta} \frac{2 \bar{E}}{\Delta \sigma_{n}} \quad \text { and } n \geq 3,
\end{aligned}
$$

where the initial iteration is defined as

$$
\frac{\Delta \sigma_{0}}{2}=\frac{M}{\frac{2 w}{3} \frac{2}{\sqrt{3}}\left(\frac{t h k}{2}\right)^{2} \frac{3}{\beta+2}} .
$$

Secondly, the conventional formulation of the R-O model (9) is applied to evaluate the total strain range $\Delta \varepsilon_{\text {tot }}$ corresponding to the equivalent stress range obtained in Eqs (16) and (17). Such a useful relation for $\Delta \varepsilon_{\text {tot }}(M)$ allows the estimation of an important control parameter of the LCF experiments, when the geometry of specimen is known and plastic deformation of a material is comprehensively described by the R-O model. Figure 3 illustrates the application of both approaches (direct by Eqs $(11-15)$ and inverted by Eqs $(9,16,17))$ to the parent material plate used in the experiments $[6,7,8,9]$ with particular dimensions of cross-section $(t h k=26 \mathrm{~mm}$ and $w=200 \mathrm{~mm}$ ) and particular material properties described by the R-O model $(E=160 \mathrm{GPa}, \nu=0.3, B=1741.96 \mathrm{MPa}, \beta=0.2996)$.

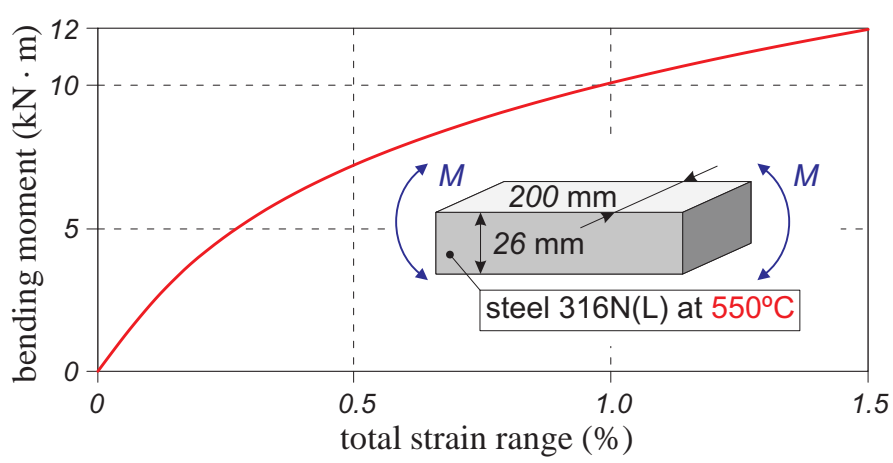

Figure 3: Curve presenting $M$ vs. $\Delta \varepsilon_{\text {tot }}$ relationship for a parent plate with particular cross-section and described by particular R-O model material constants

\subsection{Evaluation of limit load}

It is desirable to convert the absolute values of bending moment $M$ into values of normalised bending moment $\tilde{M}$, which is suitable for the formulation of an analytic assessment model for number of cycles to creep-fatigue failure $N^{\star}$, as proposed in [4]. Referring to [4] $\tilde{M}$ is defined as the relation of variable bending moment range $\Delta M$ to shakedown limit $\Delta M_{\mathrm{sh}}$ :

$$
\tilde{M}=\Delta M / \Delta M_{\mathrm{sh}},
$$

where $M_{\mathrm{sh}}$ is called initial yielding moment according to [21] and corresponds to the structural conditions, when yielding is just beginning at the edge of a beam.

The limit load and shakedown limit are evaluated with an elastic-perfectly-plastic (EPP) model and a von Mises yield condition using material properties corresponding to the saturated cyclic plasticity response $\left(E, \sigma_{\mathrm{y}}\right.$ and $\left.v\right)$ reported in Table 1 of [4] for the steel AISI type $316 \mathrm{~N}(\mathrm{~L})$ at $550^{\circ} \mathrm{C}$.

In the case of a rectangular cross-section plate in bending, assuming plane strain conditions (14), $M_{\mathrm{sh}}$ is defined analytically according to [21] as

$$
M_{\text {sh }}=\frac{\sigma_{\text {eop y } w t h k^{2}}}{6} \text { with } \quad \sigma_{\text {eop y }}=\frac{2}{\sqrt{3}} \sigma_{\mathrm{y}} .
$$

The values of bending moment exceeding $M_{\text {sh }}$ with further growth of plastic strain gradually approach the limit load value or fully plastic moment, which is defined analytically [21] as

$$
M_{\lim }=\sigma_{\text {eop y }} w t h k^{2} / 4 \text {. }
$$

When $M$ reaches the value of $M_{\text {lim }}$, it is assumed that the plate cross-section is completely in plastic flow leading to a plastic hinge and structural collapse. It should be noted that the ratio $M_{\text {lim }} / M_{\mathrm{sh}}=1.5$ changes if the cross-sectional shape is not rectangular or if a plate with rectangular cross-section contains welds. Refer to [21] for other cases of a beam crosssection. In particular case of type 1 or 2 weldments availability, the value of $M_{\text {lim }}$ remains the same, because the $\sigma_{\mathrm{y}}$ values of weld associated materials are usually higher than the $\sigma_{\mathrm{y}}$ of parent material. So plastic hinge usually happens in locations remote from weld for uniformly distributed bending moment. At least, this assumption is true for the steel AISI type $316 \mathrm{~N}(\mathrm{~L})$ at $550^{\circ} \mathrm{C}$ [4]. However, the value $M_{\text {sh }}$ for welded plate usually 
Table 3: The values of maximum normalised bending moment $\tilde{M}_{\max }$ obtained numerically and corresponding to the configurations defined in Table 1

\begin{tabular}{cl|cc}
\hline \hline \multirow{2}{*}{ No. } & \multirow{2}{*}{ Configuration } & \multicolumn{2}{|c}{$\tilde{M}_{\max }$} \\
\cline { 3 - 4 } & & type 1 & type 2 \\
\hline 1 & Perfectly dressed & 1.50906 & 1.51593 \\
2 & Typically dressed & 1.54644 & 1.55124 \\
3 & Precisely as-welded & 1.74042 & 1.78075 \\
4 & Typically as-welded & 2.02637 & 2.05556 \\
5 & Coarsely as-welded & 2.32326 & 2.30184 \\
\hline \hline
\end{tabular}

decreases, since the yielding starts at lower values of applied bending moment $M$ comparing to whole plate, because of material and geometry non-uniformity. In [4], this ratio was called the maximum normalised bending moment

$$
\tilde{M}_{\max }=\Delta M_{\text {lim }} / \Delta M_{\text {sh }},
$$

and it had a value of 1.551 for Type 2 dressed weldment [4]. Therefore, $\tilde{M}_{\max }$ is dependent on the particular geometric configuration of the weldment, and therefore should be taken into account in the formulation of parametric relations. Following this assumption and Eqs (18) and (21) the normalised bending moment is introduced in the following form:

$$
\tilde{M}=\frac{M}{M_{\mathrm{sh}}}=\frac{M \tilde{M}_{\mathrm{max}}}{M_{\mathrm{lim}}} \quad \text { with } \quad M_{\mathrm{lim}}=\frac{\sigma_{\mathrm{y}} w t h k^{2}}{2 \sqrt{3}} .
$$

Thus, the awareness of the parent material yield stress $\sigma_{\mathrm{y}}$ of the steel AISI type $316 \mathrm{~N}(\mathrm{~L})$ reported in Table 1 of [4] and geometrical parameters of specimen $(t h k=26$ and $w=200)$ allows the calculation of the limit bending moment as $M_{\mathrm{lim}}=$ $10.564[\mathrm{kN} \cdot \mathrm{m}]$ for the conditions of experiments $[6,7,8,9]$. If the weld geometry is the same as in the cruciform weldment specimens, then $\tilde{M}_{\max }=1.551$ and the values of normalised bending moment $\tilde{M}$ in experiments $[6,7,8,9]$ are calculated as reported in Table 4 of [4]. For other geometrical configurations of weldments, the set of $\tilde{M}$ will be slightly different, because $\tilde{M}_{\max }$ is individual for each geometrical configuration and were estimated numerically using step-by-step FEA.

Table 3 lists the values of $\tilde{M}_{\max }$ corresponding to the geometric configurations defined in Table 1 for type 1 and 2 weldments. These values are calculated by Eq. (21), which includes the values of $M_{\mathrm{lim}}$ and $M_{\mathrm{sh}}$ obtained numerically for each of the 10 configurations using step-by-step FEA with an EPP material model. Using the values of $M$ from Table 2, the values of $\tilde{M}_{\max }$ reported in Table 3 and the value of $M_{\text {lim }}=10.564[\mathrm{kN} \cdot \mathrm{m}]$, the values of normalised moment $\tilde{M}$ for each configuration and each $\Delta \varepsilon_{\text {tot }}$ can be calculated by applying Eq. (22). Thus, in order to provide the values of $\tilde{M}$ in fully analytical form, the values of $\tilde{M}_{\max }$ have to be defined as dependent on the geometric parameters of the weld profile ( $\alpha$ and $\beta$ ).

The maximum normalised moment $\tilde{M}_{\max 1}$ for the type 1 weldment is dependent on angle $\alpha$ as follows

$$
\begin{aligned}
& \tilde{M}_{\max 1}(\alpha)=f_{1}(\alpha)[1-H(\alpha)]+f_{2}(\alpha) H(\alpha) \quad \text { with } \\
& f_{1}(\alpha)=m_{1} \alpha+m_{2}, \quad f_{2}(\alpha)=m_{3} \alpha+m_{4} \quad \text { and } \\
& H(\alpha)=0.5+0.5 \tanh \left(\frac{\alpha-m_{5}}{m_{6}}\right) .
\end{aligned}
$$

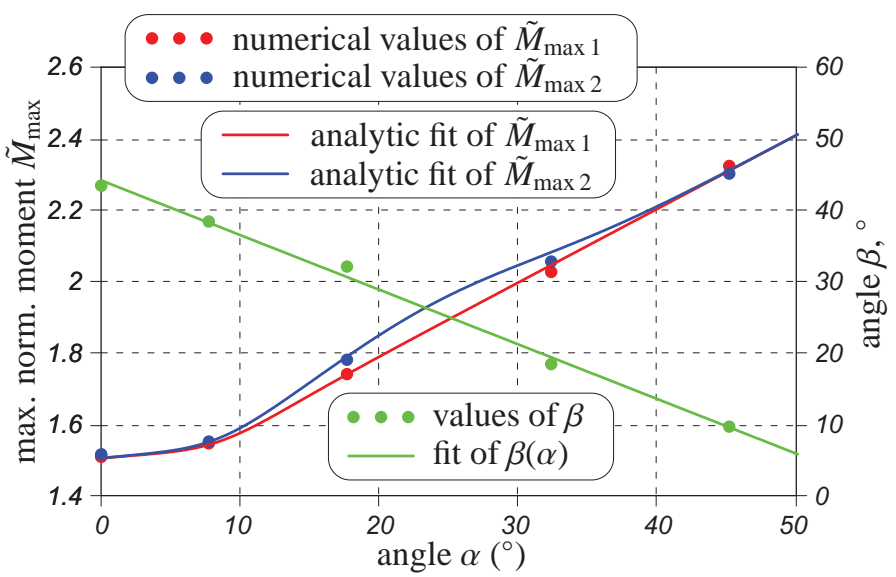

Figure 4: Numerical values of maximum normalised moment $\tilde{M}_{\max }$ from Table 3 fitted by analytic approximations (23) and (24)

In notation (23) $m_{1}=0.00483$ and $m_{2}=1.50906$ are fitting parameters of the first linear part $f_{1}(\alpha) ; m_{3}=0.02062$ and $m_{4}=1.37825$ are fitting parameters of the second linear part $f_{2}(\alpha) ; m_{5}=8.28436$ is the value of $\alpha$ corresponding to intersection of functions $f_{1}(\alpha)$ and $f_{2}(\alpha)$ and $m_{6}=5$ is the smoothing parameter in an analytic approximation $H(\alpha)$ of the Heaviside step function. The result of fitting the $\tilde{M}_{\max 1}$ numerical values from Table 3 by the analytic function $\tilde{M}_{\max 1}(\alpha)$ in the form of Eq. (23) is illustrated in Fig. 4.

Since the difference between values of $\tilde{M}_{\max }$ for types 1 and 2 corresponding to the same values of $\alpha$ is relatively small, it can be concluded that the angle $\alpha$ has a much more significant impact on the maximum normalised moment $\tilde{M}_{\max 2}$ than the angle $\beta$ for the type 2 weldment. Moreover, the effect of $\beta$ on $\tilde{M}_{\max 2}$ is limited to a quite narrow range of angles. Therefore, an optimal way to account for angle $\beta$ is to fit the difference between $\tilde{M}_{\max 2}$ and $\tilde{M}_{\max 1}$ from Table 3 with a Gaussian function dependent on $\beta$ and produce a symmetric "bell" curve. In this case, the maximum normalised moment $\tilde{M}_{\max }$ for the types 1 and 2 weldments is dependent on angles $\alpha$ and $\beta$ :

$$
\tilde{M}_{\max }(\alpha, \beta)=\tilde{M}_{\max 1}(\alpha)+m_{7} \exp \left(-m_{8}\left[\beta-m_{9}\right]^{2}\right),
$$

where $m_{7}=0.06768$ is the height of the curve's peak, $m_{8}=$ 0.01437 controls the width of the "bell", and $m_{9}=25.995$ is the position of the centre of the peak. To reduce the number of variables in Eq. (24), the angles of $\alpha$ and $\beta$ were chosen so that their values formed a linear relation

$$
\beta(\alpha)=44.1451-0.76530 \alpha .
$$

Substitution of Eq. (25) into Eq. (24) means that $\tilde{M}_{\max }$ is a function of $\alpha$ only, as illustrated in Fig. 4.

Finally, taking Eq. (20) for the bending moment $M$ and Eq. (13) for the fully plastic moment $M_{\text {lim }}$, which are both dependent on material properties $\left(E, v, B, \beta, \sigma_{\mathrm{y}}\right)$ and parameters of plate cross-section ( $w$ and thk), and Eq. (24) for the maximum normalised moment $\tilde{M}_{\max }$ dependent on parameters of weld profile ( $\alpha$ and $\beta$ ), and using them in Eq. (22) results in the fully parametric formulation of the normalised bending moment dependent on total strain range $\tilde{M}\left(\Delta \varepsilon_{\text {tot }}\right)$. 


\section{Structural integrity assessments}

\subsection{Numerical creep-fatigue evaluation}

Since the principal goal of the research is the formulation of parametric relations able to describe long-term structural integrity of weldments, the creep-fatigue strength of each of the configurations from Table 1 should be evaluated in a wide range of loading conditions. These conditions are presented by different combinations of $\Delta \varepsilon_{\text {tot }}$ in the parent plate outer fibre, as a characteristic of fatigue effects, and duration $\Delta t$ of dwell period, as a characteristic of creep effects. The set of $\Delta \varepsilon_{\text {tot }}$ values used are the same as in the experimental studies $[6,7,8,9]$, see Table 2. The set of $\Delta t$ values used are the same as in the previous simulation study [4]: 0, 0.5, 1, 2, 5, 10, 100, 1000 and 10000 hours. Therefore, for each of the 10 configurations 45 creepfatigue evaluations must be performed with different values of $\Delta \varepsilon_{\text {tot }}$ and $\Delta t$. In order to estimate 450 values of number of cycles to failure $N^{\star}, 450 \mathrm{FE}$-simulations of the parametric models shown in Fig. 2 have been carried out, using the LMM method, material models and constants given in [4]. The outputs of the LMM have been processed by the creep-fatigue procedure proposed in [4] to evaluate $N^{\star}$, because it has been successfully validated against experimental data $[6,7,8,9]$.

The concept of the proposed creep-fatigue evaluation procedure, considering time fraction rule for creep-damage assessment, is explained in detail in [4] and consists of 5 steps:

1. Estimation of saturated hysteresis loop using the LMM;

2. Estimation of fatigue damage using S-N diagrams;

3. Assessment of stress relaxation with elastic follow-up;

4. Estimation of creep damage using creep rupture curves;

5. Estimation of total damage using an interaction diagram.

Since the LMM requires lower computational effort compared to other methods, it appears to be an effective tool for express analysis of a large number of different loading cases using automation techniques. In order to perform $450 \mathrm{FE}$-simulations in CAE-system ABAQUS and effectively retrieve 450 values of $N^{\star}, 3$ analysis improvements using automation have been developed and applied in this parametric study.

The first automation technique is the embedding of all 5 steps of the proposed creep-fatigue evaluation procedure in FORTRAN code of user material subroutine UMAT containing the implementation of the LMM and material models described in [4]. For a detailed description of the numerical procedure for the creep strain and flow stress estimation in the LMM code refer to $[5,20]$, and for a general guide to the LMM implementation using the ABAQUS user subroutines refer to [19]. The creep-fatigue evaluation procedure is implemented once the LMM has converged upon the stabilised cyclic behaviour. The LMM analysis was performed using three load instances in the cycle with creep dwell: 1) end of direct loading, 2) end of dwell period, 3) end of reverse loading. This results in a saturated hysteresis loop in terms of effective strain and effective von Mises stress for each integration point in the FE-model, as shown in Fig. 5 of [4]. The most important parameters (derived in the 1st step of the procedure) for further creep-fatigue evaluation are the total strain range $\Delta \varepsilon_{\text {tot }}$, stress $\sigma_{1}$ at the beginning of dwell period and the elastic follow-up factor $Z$. These parameters from each integration point with material properties for elasticity, fatigue and creep, defined in the ABAQUS input file, are transferred into a new subroutine. This subroutine implements the next 4 steps of the procedure [4], which calculates and outputs the following parameters into ABAQUS result ODB-file: time to creep rupture $t^{*}$, creep damage accumulated per cycle $\omega_{1 \mathrm{c}}^{\mathrm{cr}}$, number of cycles to fatigue failure $N^{*}$, fatigue damage accumulated per 1 cycle $\omega_{1 \mathrm{c}}^{\mathrm{f}}$, and the most important - total number of cycles to failure in creep-fatigue conditions $N^{\star}$ obtained using the damage interaction diagram proposed by Skelton and Gandy [22]. It should be noted that this evaluation procedure was implemented in previous work [4] using Excel spreadsheets only for the most critical locations, identified manually as sites of $\Delta \varepsilon_{\text {tot }}$ and $\sigma_{1}$ maximum values.

An example of the creep-fatigue evaluation procedure outputs for the configuration no. 2 (typically dressed) of type 2 weldment corresponding to the loading case of $\Delta \varepsilon_{\text {tot }}=1 \%$ and $\Delta t=5$ hours is illustrated in Fig. 5. These results correspond to the FEA contour plots of the LMM outputs (obtained in Step 1) including $\Delta \varepsilon_{\mathrm{tot}}, \varepsilon^{\mathrm{cr}}, \varepsilon_{\mathrm{vM}}^{\mathrm{eq}}$ at the beginning of dwell and $\varepsilon_{\mathrm{vM}}^{\mathrm{eq}}$ at the end of dwell, explained in [4] and illustrated there in Fig. 9. The critical location with $N^{\star}=279$ cycles to failure for this case is the corner element in the weld toe adjacent to HAZ. The distribution of pure creep damage $\omega_{\text {cr }}$ with maximum value $\omega_{\mathrm{cr}}^{\max }=0.294$ at the critical location is shown in Fig. 5a. The distribution of pure fatigue damage $\omega_{\mathrm{f}}$ with maximum value $\omega_{\mathrm{f}}^{\max }=0.375$ at the critical location is shown in Fig. 5b. The distribution of total damage $\omega_{\text {tot }}$ with maximum value $\omega_{\text {tot }}^{\max }=0.669$ at the critical location is shown in Fig. 5c. It should be noted that value of $\omega_{\text {tot }}^{\max }$ doesn't exceed 1 , because the non-linear damage interaction diagram [22] is used in creepfatigue evaluation. The distribution of $N^{\star}$ with minimum value $N_{\text {min }}^{\star}=279$ at the critical location is shown in Fig. 5 d.

Exactly the same approach is used to demonstrate an example of a type 1 weldment comprising geometry configuration no. 2 (typically dressed) and loading case of $\Delta \varepsilon_{\text {tot }}=1 \%$ and $\Delta t=$ 5 hours. Figure 6 shows the outputs of FEA with the LMM, while Fig. 6 shows the outputs of the creep-fatigue evaluation procedure. The critical location with $N^{\star}=206$ cycles to failure for this type 1 is the same as for the type 2 weldment - the corner element in the weld toe adjacent to HAZ.

The distribution of total strain range $\Delta \varepsilon_{\text {tot }}$, with maximum value $\Delta \varepsilon_{\text {tot }}^{\max }=1.58 \%$ at the critical location, is shown in Fig. 6a. The distribution of equivalent creep strain $\varepsilon^{\mathrm{cr}}$ at load instance 2 with maximum value $\varepsilon_{\max }^{\mathrm{cr}}=2.40953 \mathrm{E}-3$ at the critical location is shown in Fig. 6b. The distribution of equivalent von Mises stress $\sigma_{\mathrm{vM}}^{\mathrm{eq}}$ at the beginning of dwell at load instance 1 with value $\sigma_{1}^{\mathrm{eq}}=334.743 \mathrm{MPa}$ at the critical location is shown in Fig. 6c. The distribution of equivalent von Mises stress $\sigma_{\mathrm{vM}}^{\mathrm{eq}}$ at the end of dwell at load instance 2 with value $\sigma_{2}^{\mathrm{eq}}=287.954 \mathrm{MPa}$ at the critical location is shown in Fig. 6d. Therefore, the drop of stress $\Delta \sigma^{\mathrm{eq}}=46.789 \mathrm{MPa}$ during $\Delta t=5$ hours of dwell provides the value of elastic follow up factor $Z=7.25$ at the critical location.

The distribution of pure creep damage $\omega_{\text {cr }}$ with maximum value $\omega_{\mathrm{cr}}^{\max }=0.323$ at the critical location is shown in Fig. 7a. 

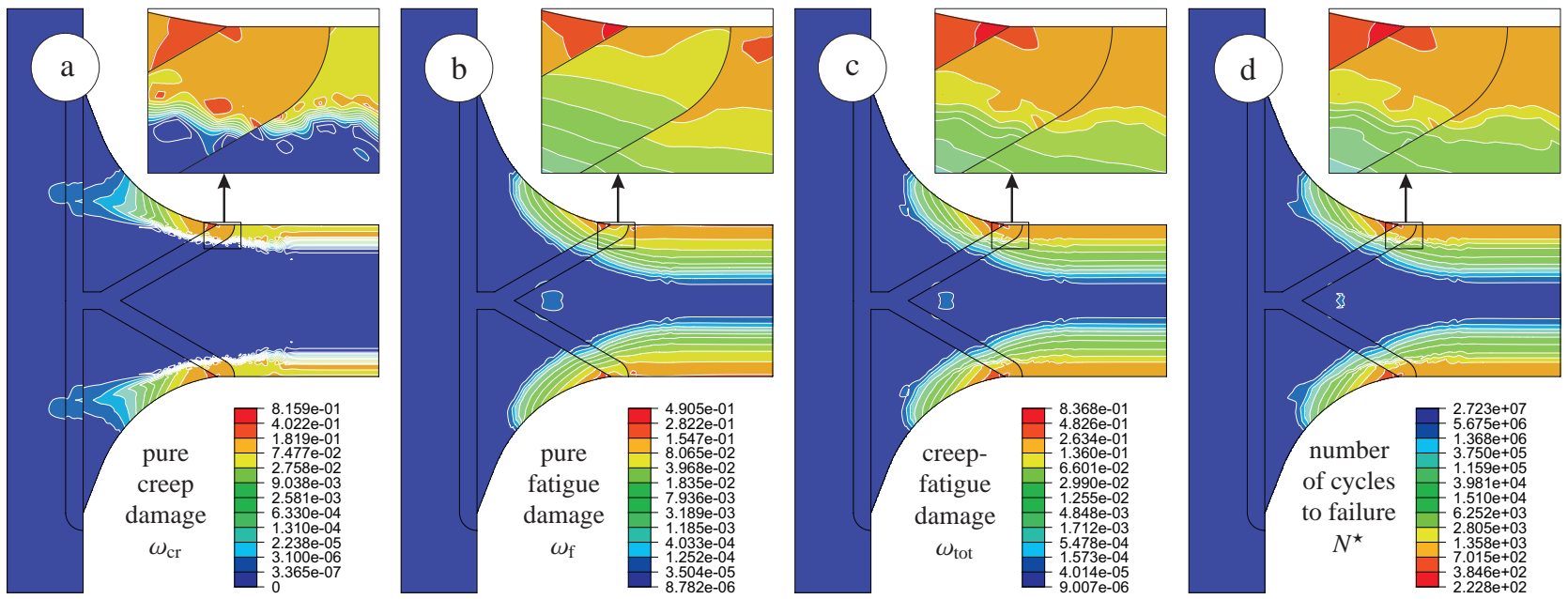

Figure 5: Contour plots of LMM results for type 2 weldment corresponding to $\Delta \varepsilon_{\text {tot }}=1 \%$ on the outer fiber and $\Delta t=5$ hours of dwell, which lead to creep-fatigue failure in weld toe after 279 cycles: a) pure creep damage $\omega_{\mathrm{cr}}$; b) pure fatigue damage $\omega_{\mathrm{f}}$; c) creep-fatigue damage $\omega_{\text {tot }}$; d) number of cycles to failure $N^{\star}$
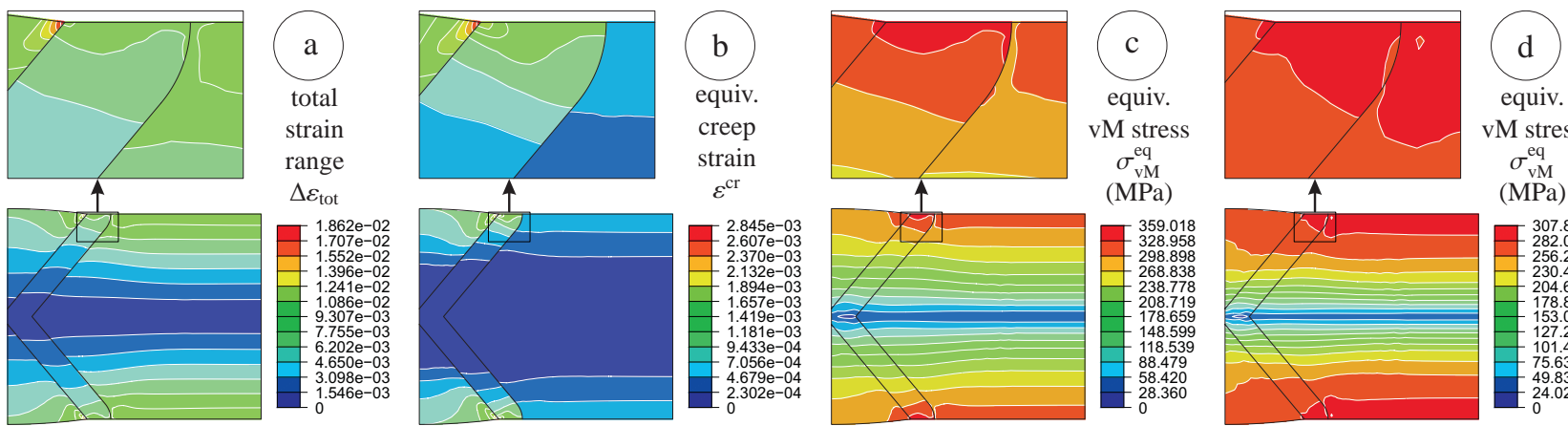

equiv.

vM stress

$\sigma_{\mathrm{vM}}^{\mathrm{eq}}$

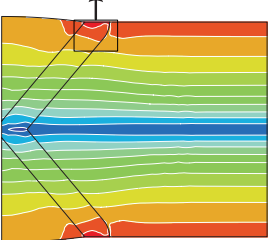

(MPa)

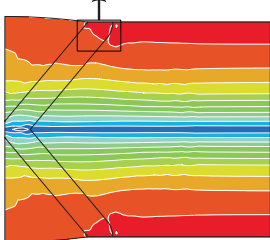

(MPa)

Figure 6: Contour plots of LMM results for type 1 weldment corresponding to $\Delta \varepsilon_{\text {tot }}=1 \%$ on the outer fiber of plate and $\Delta t=5$ hours of dwell: a) total strain range $\Delta \varepsilon_{\text {tot }}$; b) equivalent creep strain $\varepsilon^{\text {cr }}$; c) equivalent von Mises stress $\varepsilon_{\mathrm{vM}}^{\text {eq }}$ at the beginning of dwell; d) equivalent von Mises stress $\varepsilon_{\mathrm{vM}}^{\text {eq }}$ at the end of dwell
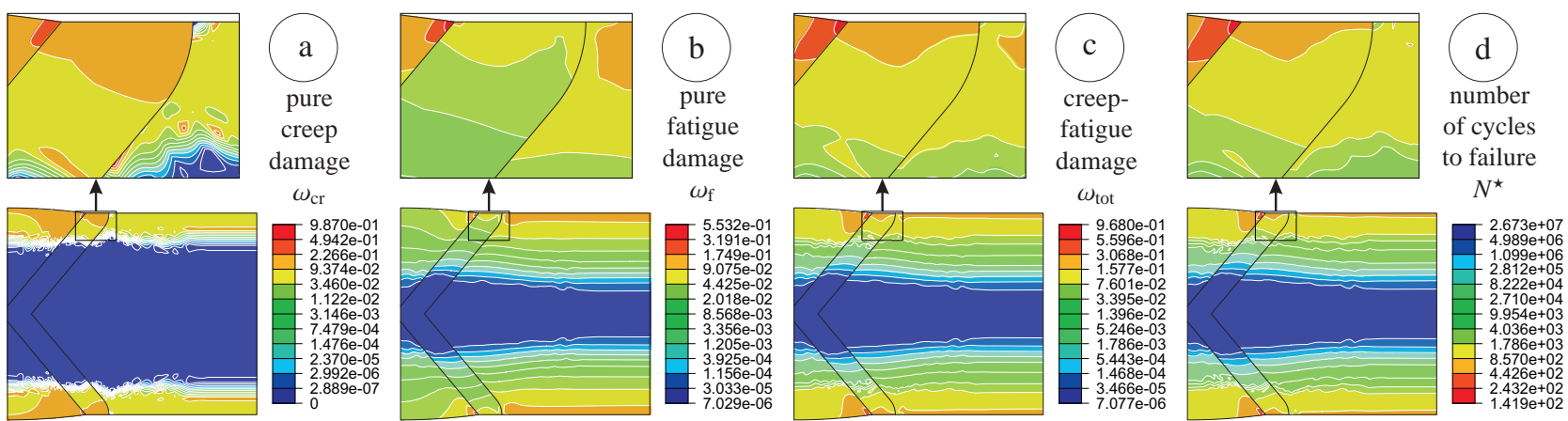

Figure 7: Contour plots of LMM results for type 1 weldment corresponding to $\Delta \varepsilon_{\text {tot }}=1 \%$ on the outer fiber and $\Delta t=5$ hours of dwell, which lead to creep-fatigue failure in weld toe after 206 cycles: a) pure creep damage $\omega_{\mathrm{cr}}$; b) pure fatigue damage $\omega_{\mathrm{f}}$; c) creep-fatigue damage $\omega_{\text {tot }}$; d) number of cycles to failure $N^{\star}$ 
The distribution of pure fatigue damage $\omega_{\mathrm{f}}$ with maximum value $\omega_{\mathrm{f}}^{\max }=0.345$ at the critical location is shown in Fig. $7 \mathrm{~b}$. The distribution of total damage $\omega_{\text {tot }}$ with maximum value $\omega_{\text {tot }}^{\max }=0.668$ at the critical location is shown in Fig. 7c. The distribution of $N^{\star}$ with minimum value $N_{\min }^{\star}=206$ at the critical location is shown in Fig. 7d.

In spite of the same critical location and almost equal values of the accumulated total damage at failure for types 1 and 2 weldments, type 1 has less residual life caused by the increased values of parameters characterising the hysteresis loop $\left(\Delta \varepsilon_{\text {tot }}\right.$, $\varepsilon^{\mathrm{cr}}, \sigma_{1}^{\mathrm{eq}}, \sigma_{2}^{\mathrm{eq}}$ and $Z$ ). Thus, one can conclude that geometrical parameter $\beta$ has a significant influence on $N^{\star}$.

The second automation technique is the development of a stand-alone application using Embarcadero Delphi integrated development environment using Delphi programming language. This simple application automatically carries out the sequence of all 45 FE-simulations with different $M$ (corresponding to $\Delta \varepsilon_{\text {tot }}$ according to Table 2) and $\Delta t$ values for each of the configurations from Table 1 . This is implemented by automated modification of the UMAT subroutine including changing of loading values ( $M$ and $\Delta t)$ and output file names, therefore producing 45 ABAQUS result ODB-files.

The third automation technique is the development of a script using ABAQUS Python Development Environment (Abaqus PDE) using Python programming language [23]. This simple script, when started in ABAQUS/CAE environment, appends the list of 45 ABAQUS result ODB-files corresponding to one configuration. For each of ODB-files, it reads the values of $N^{\star}$ in each integration point, selects the integration point with minimum value of $N^{\star}$ over the FE-model, and writes the element number, integration point number and material name to an output text file. Therefore, the critical locations and corresponding values of $N^{\star}$ are extracted automatically for all 450 configurations and loading cases. Obtained results can be used for the formulation of an analytic assessment model suitable for the fast estimation of $N^{\star}$ for a variety of loading conditions ( $\tilde{M}$ and $\Delta t)$ and geometrical weld profile parameters $(\alpha$ and $\beta$ ).

\subsection{Analytic assessment model}

For each of the 10 configurations from Table 1, the array of assessment results consisting of 45 values of $N^{\star}$ corresponding to particular values of $\tilde{M}$ and $\Delta t$ is fitted using the least squares method by the following function proposed in the form of power-law in [4]:

$$
\log \left(N^{\star}\right)=\tilde{M}^{-b(\Delta t)} / a(\Delta t),
$$

where the fitting parameters dependent on dwell period $\Delta t$ are

$$
\begin{aligned}
a(\Delta t)= & a_{3} \log (\Delta t+1)^{3}+a_{2} \log (\Delta t+1)^{2} \\
& +a_{1} \log (\Delta t+1)+a_{0} \text { and } \\
b(\Delta t)= & b_{3} \log (\Delta t+1)^{3}+b_{2} \log (\Delta t+1)^{2} \\
& +b_{1} \log (\Delta t+1)+b_{0},
\end{aligned}
$$

and the independent fitting parameters are reported in Table (4).

In order to capture all configurations with an unified set of fitting parameters, parameters $a_{0}, a_{1}, a_{2}, a_{3}, b_{0}, b_{1}, b_{2}, b_{3}$ from
Table 4 should be defined as dependent on geometric parameters $\alpha$ and $\beta$ using the least squares method. For the type 1 weldments these parameters are dependent on angle $\alpha$ only:

$$
\begin{aligned}
& a_{0}^{\mathrm{T} 1}(\alpha)=-4.175 \cdot 10^{-5} \alpha^{2}+2.72 \cdot 10^{-3} \alpha+0.227, \\
& a_{1}^{\mathrm{T} 1}(\alpha)=-2.169 \cdot 10^{-3} \alpha+1.21 \cdot 10^{-1}, \\
& a_{2}^{\mathrm{T} 1}(\alpha)=1.907 \cdot 10^{-3} \alpha-7.093 \cdot 10^{-2}, \\
& a_{3}^{\mathrm{T} 1}(\alpha)=-5.352 \cdot 10^{-4} \alpha+1.968 \cdot 10^{-2} \\
& b_{0}^{\mathrm{T} 1}(\alpha)=-4.76324 \cdot 10^{-3} \alpha+0.793, \\
& b_{1}^{\mathrm{T} 1}(\alpha)=1.42 \cdot 10^{-4} \alpha^{2}-8.547 \cdot 10^{-3} \alpha+0.4028, \\
& b_{2}^{\mathrm{T} 1}(\alpha)=1.531 \cdot 10^{-3} \alpha-0.3015, \\
& b_{3}^{\mathrm{T} 1}(\alpha)=-3.08 \cdot 10^{-4} \alpha+8.364 \cdot 10^{-2} .
\end{aligned}
$$

For the type 2 weldments these parameters include the dependence on angle $\alpha$ from Eqs (28) and an additional effect of angle $\beta$ as in the following form:

$$
\begin{aligned}
a_{0}^{\mathrm{T} 2}(\alpha, \beta)= & a_{0}^{\mathrm{T} 1}(\alpha)+3.179 \cdot 10^{-4} \beta+2.355 \cdot 10^{-3}, \\
a_{1}^{\mathrm{T} 2}(\alpha, \beta)= & a_{1}^{\mathrm{T} 1}(\alpha)-1.636 \cdot 10^{-3} \beta+3.043 \cdot 10^{-2}, \\
a_{2}^{\mathrm{T} 2}(\alpha, \beta)= & a_{2}^{\mathrm{T} 1}(\alpha)+1.636 \cdot 10^{-3} \beta-3.043 \cdot 10^{-2}, \\
a_{3}^{\mathrm{T} 2}(\alpha, \beta)= & a_{3}^{\mathrm{T} 1}(\alpha)-4.136 \cdot 10^{-4} \beta+7.33 \cdot 10^{-3}, \\
b_{0}^{\mathrm{T} 2}(\alpha, \beta)= & b_{0}^{\mathrm{T} 1}(\alpha)+0.0291 \\
& -1.684 \cdot 10^{-4} \exp (0.1622 \beta), \\
b_{1}^{\mathrm{T} 2}(\alpha, \beta)= & b_{1}^{\mathrm{T} 1}(\alpha)-0.1789, \\
b_{2}^{\mathrm{T} 2}(\alpha, \beta)= & b_{2}^{\mathrm{T} 1}(\alpha)+0.1558, \\
b_{3}^{\mathrm{T} 2}(\alpha, \beta)= & b_{3}^{\mathrm{T} 1}(\alpha)-4.546 \cdot 10^{-2} .
\end{aligned}
$$

The verification of the fit quality using the the geometrical parameters ( $\alpha$ and $\beta$ ) for the proposed relations (28) and (29) is implemented by applying Eqs (26) and (27) to estimate $N^{\star}$. Number of cycles to failure $N^{\star}$ is estimated for each of the 10 configurations using the corresponding values of angles from Table 1 and for the same load combinations as were used for the LMM analyses. The results of the verification are illustrated on diagrams in Fig. 8 for type 1 and Fig. 9 for type 2 weldments in the form of $N^{\star}$ obtained with the analytic function (26) vs. $N^{\star}$ obtained with the LMM. Comparison of the analytic and numeric $N^{\star}$ for both types of weldments shows that the quality of analytic predictions is quite close to the line of optimal match and provides a uniform scatter of results through all variants of loading conditions and configurations. The discrepancy between analytic predictions and numerical LMM outputs is generally found to be within the boundaries of an inaccuracy factor equal to 2, which is allowable for engineering analysis, producing both conservative and non-conservative results. It should be noted that $N^{\star}$ for type 1 weldments approximately belongs to the range from 10 to $10^{6}$ (see Fig. 8), while for type 2 weldments it belongs to the range from 1 to $10^{5}$ (see Fig. 9). This observation shows that type 1 weldment is less creep-fatigue resistant than type 2 weldment in the same ranges of loading conditions and manufacturing variations. This fact could be explained by the significantly smaller amount of weld and parent material used for manufacturing of type 1 weldment compared to type 2 for the same plate thickness, resulting in less rigidity and load-bearing capacity for type 1 weldment. Another important observation is that the average creep-fatigue resistivity 
Table 4: Sets of fitting parameters for Eq. (27) not dependent on $\Delta t$ corresponding to configurations from Table 1

\begin{tabular}{c|lllll|lllll}
\hline \hline \multirow{2}{*}{ Conf. } & \multicolumn{4}{|c}{ Type 1 weldment } & \multicolumn{4}{c}{ Type 2 weldment } \\
\cline { 2 - 12 } & No. 1 & No. 2 & No. 3 & No. 4 & No. 5 & No. 1 & No. 2 & No. 3 & No. 4 & No. 5 \\
\hline$a_{0}$ & 0.22459 & 0.24922 & 0.26192 & 0.26872 & 0.26584 & 0.24646 & 0.25916 & 0.27454 & 0.27947 & 0.27007 \\
$a_{1}$ & 0.11759 & 0.11152 & 0.07864 & 0.05009 & 0.02384 & 0.07922 & 0.06958 & 0.06265 & 0.04906 & 0.03958 \\
$a_{2}$ & -0.0733 & -0.0606 & -0.0281 & -0.0074 & 0.01115 & -0.0383 & -0.0196 & -0.0131 & -0.0052 & -0.0035 \\
$a_{3}$ & 0.02034 & 0.01692 & 0.00765 & 0.00151 & -0.0031 & 0.01101 & 0.00559 & 0.00352 & 0.00083 & 0.00032 \\
$b_{0}$ & 0.77482 & 0.76997 & 0.72078 & 0.63676 & 0.57224 & 0.59539 & 0.71263 & 0.72463 & 0.66209 & 0.60055 \\
$b_{1}$ & 0.39622 & 0.35439 & 0.29853 & 0.26549 & 0.31070 & 0.38309 & 0.16595 & 0.11959 & 0.09628 & 0.06507 \\
$b_{2}$ & -0.3080 & -0.2892 & -0.2725 & -0.2349 & -0.2455 & -0.2711 & -0.1207 & -0.1161 & -0.0924 & -0.0630 \\
$b_{3}$ & 0.08473 & 0.08028 & 0.07884 & 0.07130 & 0.07134 & 0.06572 & 0.02987 & 0.03439 & 0.03033 & 0.02533 \\
\hline \hline
\end{tabular}

of configuration no. 1 (perfectly dressed) is relatively the highest among all configurations for both types of weldments. The average resistivity is slightly reducing from one configuration to another with the growth of angle $\alpha$ value as shown in Figs 8 and 9 , resulting in the minimum average $N^{\star}$ for the configuration no. 5 (coarsely as-welded).

Having defined the number of cycles to failure $N^{\star}$ by Eq. (26), the residual service life in years is therefore dependent on the duration of 1 cycle, which consists of dwell period $\Delta t$ and relatively short time of deformation as follows:

$$
L^{\star}=N^{\star}\left[\frac{\Delta t}{365 \cdot 24}+\frac{2 \Delta \varepsilon_{\mathrm{tot}}(\tilde{M})}{\dot{\varepsilon}(365 \cdot 24 \cdot 60 \cdot 60)}\right],
$$

where $\dot{\varepsilon}=0.03 \% / \mathrm{s}$ is a strain rate according to experimental conditions $[6,7,8,9]$, and the parametric analytical relations for $\Delta \varepsilon_{\text {tot }}(\tilde{M})$ are derived in Sect. 3. These relations consist of Eqs (9), (16) and (17) given in Sect. 3.1 to evaluate $\Delta \varepsilon_{\text {tot }}(\Delta \sigma(M))$, where $M$ is replaced by $\tilde{M}$ and $M_{\text {lim }}$ using Eq. (22) and $\tilde{M}_{\max }$ using Eq. (24) given in Sect. 3.2. The aforementioned group of equations for the relation $\Delta \varepsilon_{\text {tot }}(\tilde{M})$ include the geometrical parameters of parent plate cross-section (thk and $w$ ) and weld profile $(\alpha$ and $\beta)$, and parent plate material parameters $(E, v$, $\left.B, \beta, \sigma_{\mathrm{y}}\right)$. This group of equations (9), (16), (17), (22) and (24) replaces Eq. (35) from [4], which is suitable for only one particular variant of weldment (type 2), weld profile (conf. 2 typically dressed) and parent plate cross-section $[6,7,8,9]$.

\section{Parametric formulation of FSRF}

Since the function $N^{\star}(\tilde{M}, \Delta t)$ proved its validity in the previous subsection, it can be applied for the fast creep-fatigue assessments of new welded structures during the design stage. However, it is generally hard to generate conclusions about the service conditions $(\tilde{M}, \Delta t)$ required to estimate particular value of $N^{\star}$. Loading conditions comprise a wide range of mechanical loading described by $\tilde{M}$ or corresponding range of $\Delta \varepsilon_{\text {tot }}$ in parent material adjacent to welded joints. Thus, introduction of a Fatigue Strength Reduction Factor (FSRF) allows a wide range of mechanical loading relevant to application area of a designed welded structure to be captured. The FSRF takes into account the difference in behaviour of the weldment compared to the parent material, considering weldments to be composed of parent material. The FSRF is determined experimentally by comparing the fatigue failure data of the welded specimen with the fatigue curve derived from tests on the parent plate material.

The current approach in R5 Volume 2/3 Procedure [10] operates with the fixed values of FSRF for 3 different types of weldments taking into account dressed and as-welded variants, which consider only the reduction of fatigue strength of weldments compared to the parent material. For austenitic steel weldments $[24,25]$, FSRF $=1.5$ is prescribed for both variants of type 1, and FSRF $=1.5$ for type 2 dressed and FSRF $=$ 2.5 for as-welded variant. All this variety of the FSRFs is representative of the reduction in fatigue endurance caused by the local strain range $\varepsilon_{\text {tot }}$ enhancement in the weldment region due to the material discontinuity and geometric strain concentration effects. The introduction of FSRF as dependent on $\Delta t$ in [4] using function $N^{\star}(\tilde{M}, \Delta t)$ for the case of type 2 dressed weldment allowed the influence of creep to be taken into account, and to provide the adjusted values of FSRF for the real operation conditions, where creep-fatigue interaction takes place. Therefore, the same approach [4] is applied to obtain $\Delta t$-dependent FSRFs for a variety of geometrical configurations considering additional dependence on parameters of weld profile ( $\alpha$ and $\beta$ ).

For this purpose Eq. (26) is converted analytically to the relation $\tilde{M}\left(N^{\star}, \Delta t\right)$ and inserted into the group of relations $\Delta \varepsilon_{\text {tot }}(\tilde{M})$ given in the end of previous subsection, resulting in the relation $\Delta \varepsilon_{\text {tot }}\left(N^{\star}, \Delta t, \alpha, \beta\right)$. This relation describes the $\Delta \varepsilon_{\text {tot }}$ in the parent material remote from weldment corresponding to particular values of $N^{\star}$ and $\Delta t$ for a particular geometrical configuration of weldment defined by $\alpha$ and $\beta$. Thus, the FSRFs, appropriate to varying values of $\Delta t$ and equal values of $N^{\star}$, are defined by the relation between the $\mathrm{S}-\mathrm{N}$ diagram corresponding to fatigue failures of parent material plate and $\mathrm{S}-\mathrm{N}$ diagrams for a weldment defined by $\alpha$ and $\beta$ :

$$
\operatorname{FSRF}=\Delta \varepsilon_{\text {tot }}^{\mathrm{par}}\left(N^{\star}\right) / \Delta \varepsilon_{\mathrm{tot}}\left(N^{\star}, \Delta t, \alpha, \beta\right),
$$

where the $\mathrm{S}-\mathrm{N}$ diagram for parent material plate is defined as

$$
\log \left(\Delta \varepsilon_{\text {tot }}^{\mathrm{par}}\right)=p_{0}+p_{1} \log \left(N^{*}\right)+p_{2} \log \left(N^{*}\right)^{2},
$$

with the following polynomial coefficients referring to [25]: $p_{0}=2.2274, p_{1}=-0.94691$ and $p_{2}=0.085943$.

The FSRFs estimated by Eq. (31) corresponding to the range of $\Delta t \in\left[0 \ldots 10^{5}\right]$ hours are defined in some particular range of 


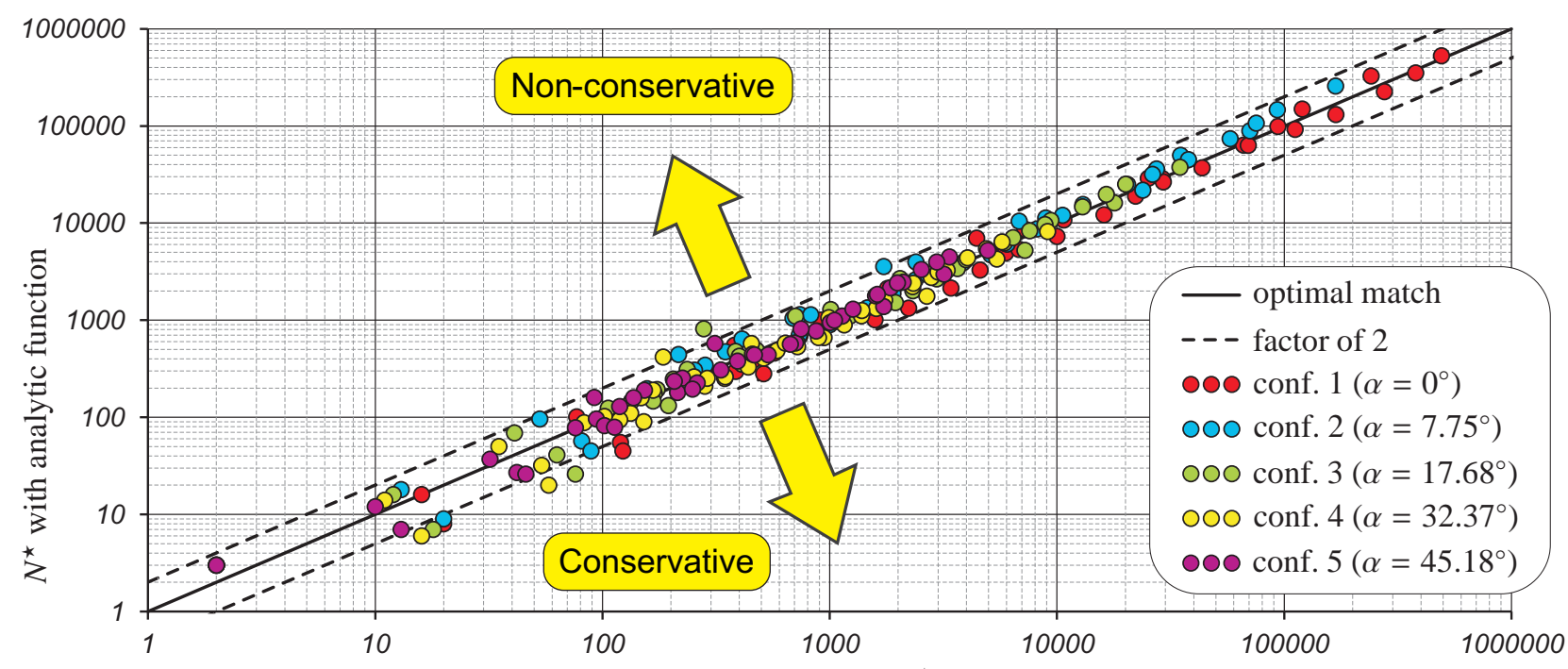

Number of cycles to failure $N^{\star}$ with the LMM

Figure 8: Comparison of number of cycles to failure $N^{\star}$ obtained with the LMM and the analytic function (26) for type 1 weldment

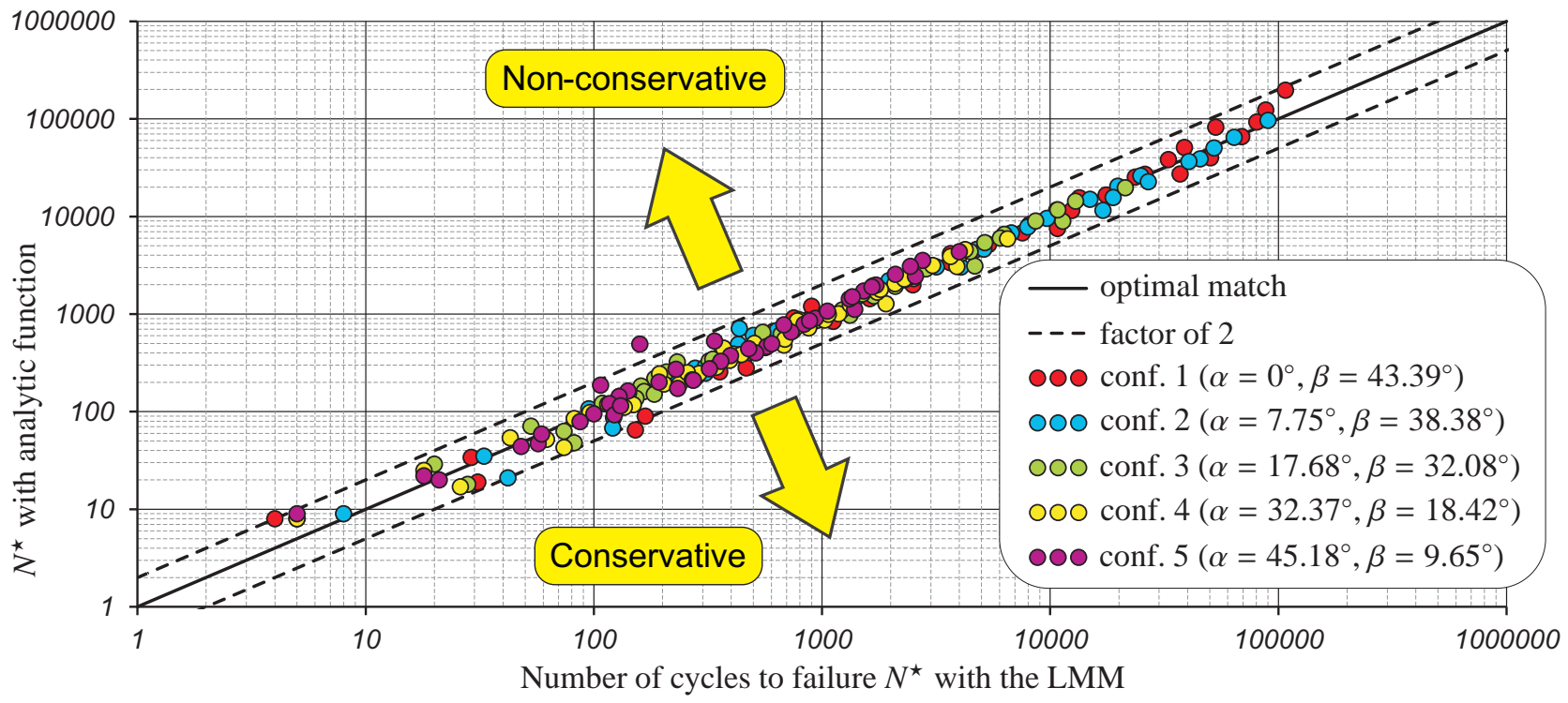

Figure 9: Comparison of number of cycles to failure $N^{\star}$ obtained with the LMM and the analytic function (26) for type 2 weldment
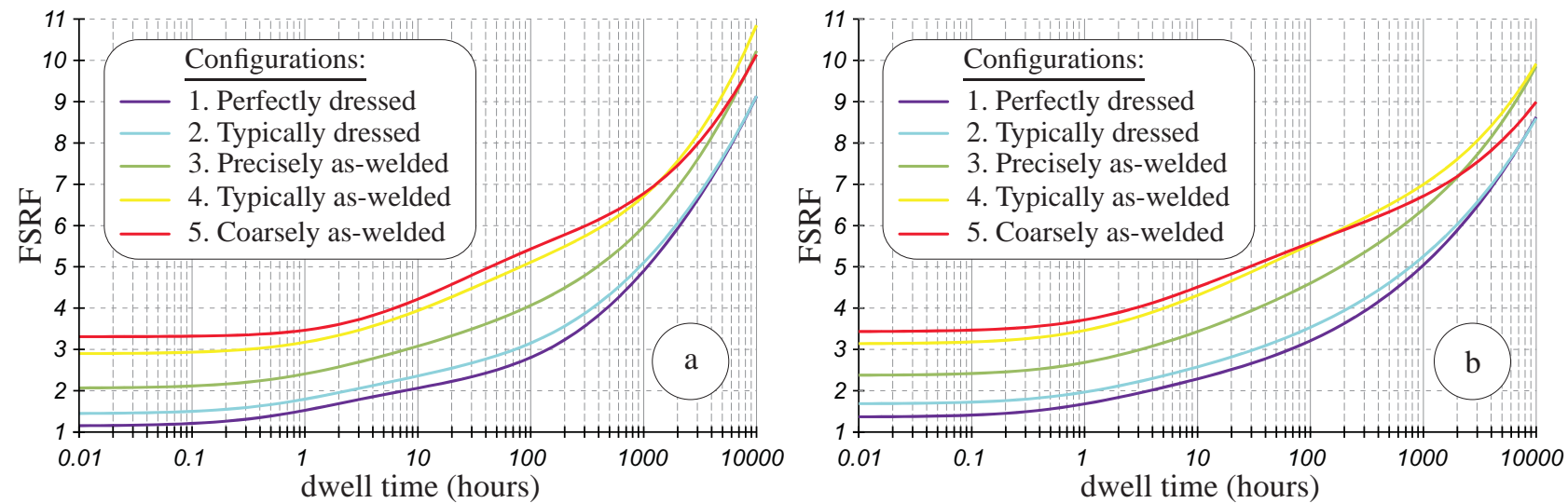

Figure 10: Dependence of FSRF on duration of dwell period $\Delta t$ for (a) type 1 and (b) type 2 weldments corresponding to the configurations from Table 1 
Table 5: The values of FSRFs for pure fatigue for types 1 and 2 weldments corresponding to the configurations from Table 1

\begin{tabular}{c|ccccc}
\hline \hline Conf. & 1 & 2 & 3 & 4 & 5 \\
\hline Type 1 & 1.146 & 1.444 & 2.062 & 2.896 & 3.308 \\
Type 2 & 1.362 & 1.682 & 2.372 & 3.137 & 3.430 \\
\hline \hline
\end{tabular}

$N^{\star}$. This range is different for each value of $\Delta t$ characterised by reducing value of the average $N^{\star}$ with the growth of $\Delta t$. The upper bound of the $N^{\star}$ range is governed by the mathematical upper limit of the S-N diagram $\Delta \varepsilon_{\text {tot }}^{\mathrm{par}}\left(N^{\star}\right)$ for parent material plate, which is defined in [4] as $\log \left(N_{\max }^{\star}\right)=p_{1} /\left(2 p_{2}\right)=5.51$ or $\Delta \varepsilon_{\text {tot }}^{\text {par }}\left(10^{5.51}\right)=0.416 \%$. The lower bound of the $N^{\star}$ range is flexible and governed by $\Delta t$ using the following function:

$$
\log \left(N_{\text {min }}^{\star}\right)=3-0.5 \log (\Delta t+1)
$$

Finally, for each of the 10 configurations from Table 1 the FSRF is defined as a continuous function of $\Delta t$ using Eq. (31) using simple averaging procedure over a dynamic range of $N^{\star}$ from $\log \left(N_{\text {min }}^{\star}\right)$ to $\log \left(N_{\text {max }}^{\star}\right)$ with step 0.01. The resultant dependencies of FSRFs on $\Delta t$ are illustrated in Fig. 10a for type 1 and in Fig. 10b for type 2 weldments with designation of different configurations. First of all, these figures show significant enhancement of FSRF for dwells $\Delta t>0.1$ hour caused by creep, which is important for design applications. The initial values of FSRFs corresponding to pure fatigue conditions $(\Delta t=0)$ are listed in Table 5 and could be compared with the values recommended in R5 Volume 2/3 Procedure [10].

The FSRF for type 1 dressed weldments is within the range 1.146-1.444 depending on the quality of grinding, while R5 gives the value 1.5 (refer to $[24,25]$ ), which is more conservative. The FSRF for type 1 precisely welded joints without grinding is within the range 1.444-2.062 depending on the quality of welding, while R5 gives the same value 1.5 , which is non-conservative. The FSRF for type 1 coarsely welded joints without any additional treatment may reach up to 3.308 , while R5 doesn't give any value for this case.

The FSRF for type 2 dressed weldments is within the range $1.362-1.682$ depending on the quality of grinding, while R5 gives the value 1.5 , which approximately corresponds to average value for the obtained range. The FSRF for type 2 precisely welded joints without grinding is within the range 1.682-2.372 depending on the quality of welding, while R5 gives the value 2.5 , which is more conservative. The FSRF for type 2 coarsely welded joints without any additional treatment may reach up to 3.43, while R5 doesn't give any value for this case.

Using the proposed approach in this work, the values of FSRFs reported in Table 5 could be easily revised, if the ranges of angles $\alpha$ and $\beta$ characterising the quality of weldment are modified. It should be noted that the FSRF of 1.682 for type 2 dressed weldment revises the value of 1.77 reported in previous work [4], because the form of fitting functions (26) and (27) has been improved in this work providing less conservatism in $N^{\star}$ predictions for pure fatigue.

\section{Conclusions}

The parametric study on creep-fatigue strength of the steel AISI type $316 \mathrm{~N}(\mathrm{~L})$ weldments of types 1 and 2 according to classification of R5 Vol. 2/3 Procedure [10] at $550^{\circ} \mathrm{C}$ has been implemented using the LMM. The study is based upon the latest developed creep-fatigue evaluation procedure [4] considering time fraction rule for creep-damage assessment. This procedure has been successfully validated in [4] against experimental data $[6,7,8,9]$ comprising reverse bending tests of cruciform weldments for different combination of loading conditions (dwell period $\Delta t$ and normalised bending moment $\tilde{M}$ ).

Parametric models of geometry and FE-meshes for both types of weldments shown in Figures 1 and 2 are developed in a way which allows variation of parameters governing shape of the weld profile (angles $\alpha$ and $\beta$ ) and loading conditions ( $\Delta t$ and $\tilde{M}$ ). Five configurations, characterised by individual sets of parameters listed in Table 1, are proposed to present different fabrication cases and to characterise weldment manufacturing quality. For each of configuration, the total number of cycles to failure $N^{\star}$ in creep-fatigue conditions is assessed numerically for different loading cases using several LMM-analysis automation techniques described in Sect. 4.1. The obtained set of $N^{\star}$ is extrapolated by the analytic function (26) dependent on $\tilde{M}$ with fitting functions (27) dependent on $\Delta t$, which includes the fitting parameters (28) and (29) dependent on geometrical parameters $(\alpha$ and $\beta)$. The difference in analytical predictions compared to LMM-based assessment is that the results for pure fatigue are relatively conservative, but are still within the factor of 2 allowed by engineering standards, as shown in in Fig. 11.

Proposed function (26) for $N^{\star}$ shows good agreement with numerical results obtained by the LMM in Figures 8 and 9 for types 1 and 2 weldments correspondingly. The discrepancy between analytic predictions and numerical LMM outputs is generally found to be within the boundaries of an inaccuracy factor equal to 2 , which is allowable for engineering analysis, producing both conservative and non-conservative results. Therefore, it is used for the identification of FSRFs intended for design purposes and dependent on $\Delta t$ and geometrical parameters ( $\alpha$ and $\beta$ ). The proposed function for FSRFs (31) is applied to all 10 configuration from Table 1 characterised by $\alpha$ and $\beta$ in order to obtain continuous dependencies on $\Delta t$, which are shown in Figures 10a and $10 \mathrm{~b}$ for types 1 and 2 weldments respectively. Therefore, this approach improves upon existing design techniques, e.g. in R5 Procedure [10], by considering the significant influence of creep. Moreover, the obtained FSRFs for pure fatigue revises the values recommended in R5 Procedure [10] removing the redundant conservatism for type 1 dressed weldments and type 2 undressed weldments.

Finally, in order to conclude about the global sensitivity of creep-fatigue strength to a change of parameters, the set of equations (26) - (29) for $N^{\star}(\tilde{M}, \Delta t, \alpha, \beta)$ are applied to create a set of contour plots shown in Fig. 11. These plots characterise the influence of geometric parameters $(\alpha$ and $\beta)$ on $N^{\star}$ at 4 different combinations of loading conditions $(\Delta t$ and $\tilde{M})$ for type 2 weldment. The global tendency is that $\alpha$ generally decreases the strength, while $\beta$ generally increases it. However 


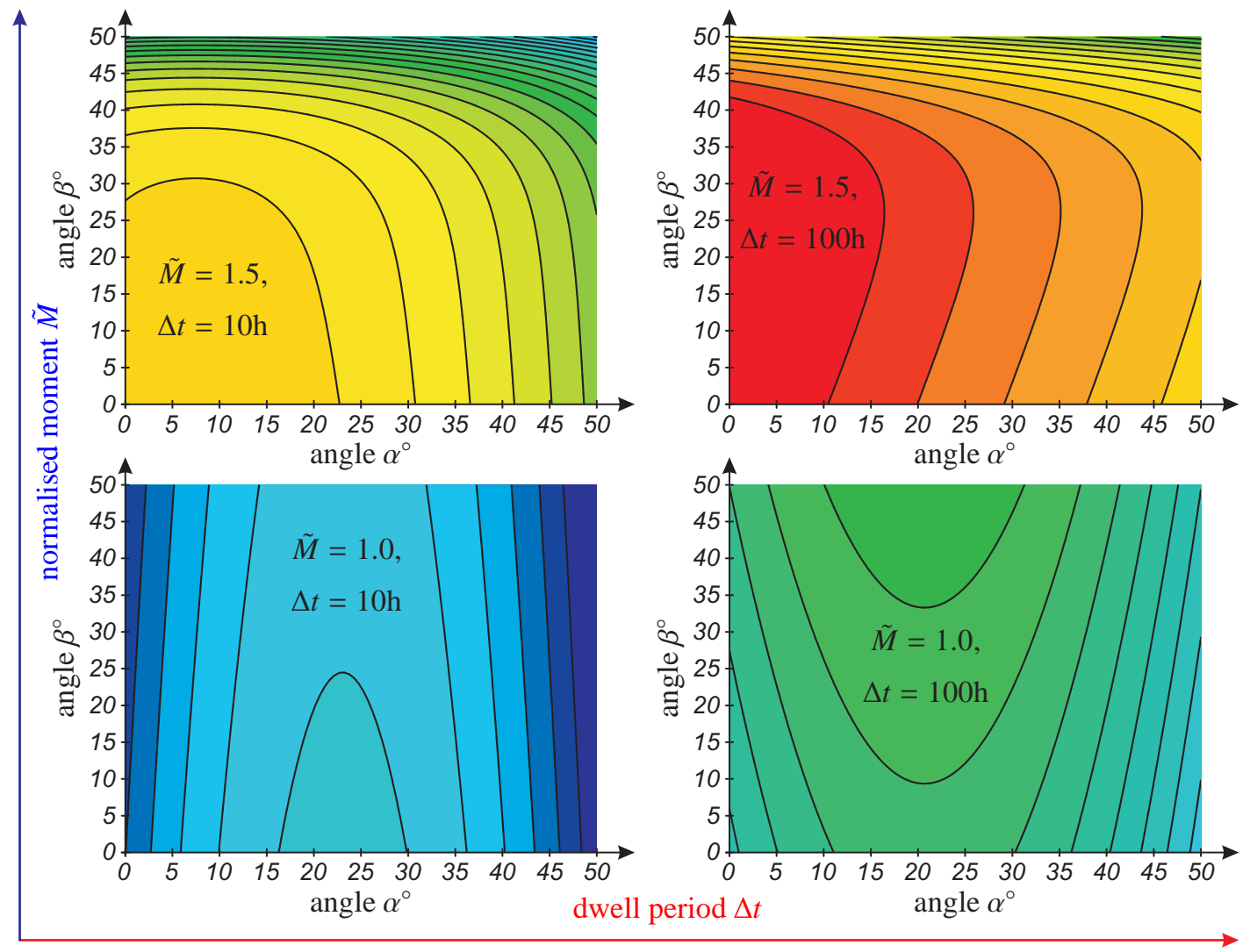

cycles to failure $N^{\star}$

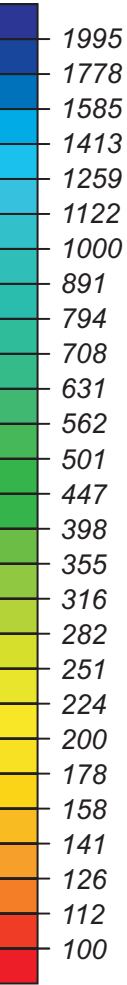

Figure 11: Contour plots for type 2 weldment characterising the influence of geometric parameters $(\alpha$ and $\beta)$ on number of cycles to failure $N^{\star}$ for different combinations of loading conditions $(\Delta t$ and $\tilde{M})$ obtained with Eqs (26) - (29)

these effects are dependent on intensity of mechanical load $\tilde{M}$ and length of dwell period $\Delta t$. The growth of $\Delta t$ changes the positive influence of $\beta$ to negative and smoothes the negative influence of $\alpha$ on $N^{\star}$. The growth of $\tilde{M}$ changes the negative influence of $\alpha$ to positive and smoothes the positive influence of $\beta$ on $N^{\star}$. The intensity of a parameter ( $\alpha$ or $\beta$ ) influence is characterised by the relative density of contour edges crossing the corresponding axis. Since both parameters can not increase their values simultaneously, only half of each plot, including upper left, lower left and lower right corners, is of importance. Figure 11 shows that the change of both loading parameters $(\Delta t$ and $\tilde{M}$ ) quite significantly changes the location of contour edges, and therefore the contribution of $\alpha$ and $\beta$ on $N^{\star}$.

Further research is devoted to parametric study on creepfatigue strength of Type 3 weldment, which includes the variable distance between welded parts $l$ as the 3rd geometric parameter along with $\alpha$ and $\beta$. The function for $N^{\star}$ should be extended to account for the effect of $l$ based upon the numerical results using LMM for different configurations. This will allow consideration of the effect of $l$ on the $\Delta t$-dependent FSRF for Type 3 dressed and as-welded variants, which has the value of 3.2 for pure fatigue prescribed in R5 Vol. 2/3 Procedure [10].

\section{Acknowledgements}

The authors deeply appreciate the Engineering and Physical Sciences Research Council (EPSRC) of the UK for the financial support in the frames of research grant no. EP/G038880/1, the University of Strathclyde for hosting during the course of this work, and EDF Energy for the experimental data.

\section{References}

1. Lee, Y.-L., Barkey, M.E., Kang, H.-T.. Metal Fatigue Analysis Handbook: Practical Problem-Solving Techniques for Computer-Aided Engineering. Oxford: Butterworth-Heinemann; 2012.

2. Radaj, D., Sonsino, C.M., Fricke, W.. Fatigue Assessment of Welded Joints by Local Approaches. Cambridge: Woodhead Publishing Limited; 2nd ed.; 2006.

3. Łagoda, T.. Lifetime Estimation of Welded Joints. Berlin: SpringerVerlag; 2008.

4. Gorash, Y., Chen, H.. Creep-fatigue life assessment of cruciform weldments using the linear matching method. Int $J$ of Pressure Vessels $\mathcal{E}$ Piping 2012;:14 p., Manuscript no. IPVP3257, in press, DOI: 10.1016/j.ijpvp.2012.12.003, https://docs.google.com/open?id=0Bx4lucS7z9cpNC1ZX2V3REk0em8.

5. Chen, H.F., Chen, W., Ure, J.. Linear matching method on the evaluation of cyclic behaviour with creep effect. In: Proc. ASME Pressure Vessels $\mathcal{E}$ Piping Conf. (PVP2012). Toronto, Canada: ASME; 2012, July 15-19.

6. Bretherton, I., Knowles, G., Slater, I.J., Yellowlees, S.F.. The fatigue and creep-fatigue behaviour of $26 \mathrm{~mm}$ thick type $316 \mathrm{~L}(\mathrm{~N})$ welded cruciform joints at $550^{\circ} \mathrm{C}$ : An interim report. Report for Nuclear Electric Ltd no. R/NE/432; AEA Technology plc; Warrington, UK; 1998.

7. Bretherton, I., Knowles, G., Bate, S.K.. PC/AGR/5087: The fatigue and creep-fatigue behaviour of welded cruciform joints: A second interim report. Report for British Energy Generation Ltd no. AEAT-3406; AEA Technology plc; Warrington, UK; 1999.

8. Bretherton, I., Budden, P.J.. Assessment of creep-fatigue endurance of large cruciform weldments. In: Trans. 15th Int. Conf. on Structural Me- 
chanics in Reactor Technology; SMiRT15 - F05/2. Seoul, Korea: IASMiRT; 1999, p. 185-192.

9. Bretherton, I., Knowles, G., Hayes, J.-P., Bate, S.K., Austin, C.J.. PC/AGR/5087: Final report on the fatigue and creep-fatigue behaviour of welded cruciform joints. Report for British Energy Generation Ltd no. RJCB/RD01186/R01; Serco Assurance; Warrington, UK; 2004.

10. Ainsworth, R.A., editor. R5: An Assessment Procedure for the High Temperature Response of Structures. Procedure R5: Issue 3. British Energy Generation Ltd, Gloucester, UK; 2003.

11. Nguyen-Tajan, T.M.L., Pommier, B., Maitournam, H., Houari, M., Verger, L., Du, Z.Z., et al. Determination of the stabilized response of a structure undergoing cyclic thermal-mechanical loads by a direct cyclic method. In: Proc. 16th Annual ABAQUS Users' Conference. Munich, Germany: Dassault Systèmes Simulia Corp.; 2003, June 4-6.

12. ABAQUS Analysis User's Manual. Dassault Systèmes Simulia Corp.; Version 6.10 ed.; 2010.

13. Ponter, A.R.S., Chen, H.F.. A minimum theorem for cyclic load in excess of shakedown, with application to the evaluation of a ratchet limit. European Journal of Mechanics - A/Solids 2001;20(4):539-553.

14. Chen, H.F., Ponter, A.R.S.. A method for the evaluation of a ratchet limit and the amplitude of plastic strain for bodies subjected to cyclic loading. European Journal of Mechanics - A/Solids 2001;20(4):555-571.

15. Chen, H.F.. Lower and upper bound shakedown analysis of structures with temperature-dependent yield stress. Journal of Pressure Vessel Technology 2010;132(1):011202:1-8.

16. Chen, H.F., Ponter, A.R.S.. A direct method on the evaluation of ratchet limit. Journal of Pressure Vessel Technology 2010;132(4):041202:1-8.

17. Chen, H.F., Ponter, A.R.S.. Linear matching method on the evaluation of plastic and creep behaviours for bodies subjected to cyclic thermal and mechanical loading. International Journal for Numerical Methods in Engineering 2006;68(1):13-32.

18. Ponter, A.R.S., Chen, H.F.. Modeling of the behavior of a welded joint subjected to reverse bending moment at high temperature. Journal of Pressure Vessel Technology 2007;129(2):254-261.

19. Tipping, D.J.. The Linear Matching Method: A Guide to the ABAQUS User Subroutines. Generic Report no. E/REP/BBGB/0017/GEN/07; Central Engineering Support; British Energy Generation Ltd, Gloucester, UK; 2008.

20. Ure, J., Chen, H., Tipping, D.. Development and implementation of the ABAQUS subroutines and plug-in for routine structural integrity assessment using the Linear Matching Method. In: Proc. SIMULIA Regional User Meeting. Manchester, UK: Dassault Systèmes Simulia Corp.; 2012, September 25-27.

21. Dowling, N.E.. Mechanical Behavior of Materials: Engineering Methods for Deformation, Fracture, and Fatigue. Upper Saddle River, USA: Pearson Prentice Hall; 3rd ed.; 2007.

22. Skelton, R.P., Gandy, D.. Creep-fatigue damage accumulation and interaction diagram based on metallographic interpretation of mechanisms. Materials at High Temperatures 2008;25(1):27-54.

23. ABAQUS Scripting User's Manual. Dassault Systèmes Simulia Corp.; Version 6.10 ed.; 2010.

24. Dean, D.W.. Recent developments in the R5 procedures for assessing the high temperature response of structures. Materials at High Temperatures 2011;28(2):95-102.

25. Bate, S.K., Hayes, J.-P., Hooton, D.G., Smith, N.G.. Further analyses to validate the R5 volume $2 / 3$ procedure for the assessment of austenitic weldments. Report for British Energy Generation Ltd no. SA/EIG/11890/R002; Serco Assurance; Warrington, UK; 2005.

\section{Nomenclature}

\section{Abbreviations}

EPP Elastic-perfectly-plastic

FEA Finite Element Analysis

FSRF Fatigue strength reduction factor

HAZ Heat-affected zone

LCF Low-cycle fatigue

LMM Linear Matching Method
MMA Manual Metal Arc

R-O Ramberg-Osgood

Variables, Constants

$\sigma$

$\Delta \sigma$

$\sigma_{\text {eop }}$

$\varepsilon$

$\dot{\varepsilon}$

$\tilde{\varepsilon}$

$\Delta \varepsilon$

$\omega$

$t$

$\Delta t$

E

$\bar{E}$

$\mu$

$N$

$L$

Z

$M$

$\tilde{M}$

$\Delta M$

$P$

$I_{X}$

$w$, thk

$\alpha, \beta$

$R_{1}, R_{2}$

$\delta$

$D$

$h_{1}, d_{1}, h_{2}, d_{2}$

$\sigma_{\mathrm{y}}$

$B, \beta$

$p_{0}, p_{1}, p_{2}$

$a_{0}, \ldots, a_{3}, b_{0}, \ldots, b_{3}$

$m_{1}, \ldots, m_{9}$

stress

stress range

edge-of-plate stress

strain

strain rate

ratio between plastic and elastic strains

strain range

damage parameter

time

dwell period

Young's (elasticity) modulus

effective elastic modulus

Poisson's ratio

number of cycles

residual life

elastic follow-up factor

bending moment

normalised moment

moment range

normal pressure

area moment of inertia

width and thickness of plate

angles governing the form of weld profile

radiuses of weld profile for type 1 and type

2 weldments correspondingly

height of weld profile in type 1 weldment

distance between opposite weld surfaces in type 2 weldment

auxiliary geometrical parameters for type

1 and type 2 weldments correspondingly

yield stress

R-O model constants

coefficients for parent material S-N curve

fitting parameters for $N^{\star}$

fitting parameters for $\tilde{M}_{\max }$

Subscripts, Superscripts

$0 \quad$ corresponding to initial value

cr creep

f fatigue

el elastic

pl plastic

* corresponding to pure fatigue

$\star \quad$ corresponding to creep-fatigue

vM von Mises

eq equivalent

tot total

1c per 1 cycle

lim corresponding to limit load

sh corresponding to shakedown limit

parent corresponding to parent material

T1 corresponding to type 1 weldment

T2 corresponding to type 2 weldment 